\title{
Automatic structures of bounded degree revisited
}

\author{
Dietrich Kuske and Markus Lohrey* \\ Universität Leipzig, Institut für Informatik, Germany \\ \{kuske, lohrey\}@informatik. uni-leipzig.de
}

\begin{abstract}
The first-order theory of a string automatic structure is known to be decidable, but there are examples of string automatic structures with nonelementary first-order theories. We prove that the first-order theory of a string automatic structure of bounded degree is decidable in doubly exponential space (for injective automatic presentations, this holds even uniformly). This result is shown to be optimal since we also present a string automatic structure of bounded degree whose first-order theory is hard for 2EXPSPACE. We prove similar results also for tree automatic structures. These findings close the gaps left open in [24] by improving both, the lower and the upper bounds.
\end{abstract}

\section{Introduction}

The idea of an automatic structure goes back to Büchi and Elgot who used finite automata to decide, e.g., Presburger arithmetic [11]. Automaton decidable theories [14] and automatic groups [12] are similar concepts. A systematic study was initiated by Khoussainov and Nerode [16] who also coined the name "automatic structure" (we prefer the term "string automatic structures" in this paper). In essence, a structure is string automatic if the elements of the universe can be represented as strings from a regular language (an element can be represented by several strings) and every relation of the structure can be recognized by a finite state automaton with several heads that proceed synchronously. String automatic structures received increasing interest over the last years [5/17/15/3/18/20/1/23/21/27/2]. One of the main motivations for investigating string automatic structures is that their first-order theories can be decided uniformly (i.e., the input is a string automatic presentation and a first-order sentence). But even the non-uniform first-order theory is far from efficient since there exist string automatic structures with a nonelementary first-order theory. This motivates the search for subclasses of string automatic structures whose first-order theories are elementary. The first such class was identified by the second author in 24] who showed that the first-order theory of every string automatic structure of bounded degree can be decided in triply exponential alternating time with linearly many alternations. A structure has bounded degree, if in its Gaifman graph, the number of neighbors of a node is bounded by some fixed constant. The paper 24] also presents a specific example of a string automatic structure of bounded degree, where the first-order theory is hard for doubly exponential alternating time with linearly many alternations. Hence, an exponential gap between the upper and lower bound remained. An upper bound of 4-fold exponential alternating time with linearly many alternations was shown for tree automatic structures (which are defined analogously to automatic structures using tree automata) of bounded degree. Our paper [22] proves a triply exponential space bound for the first-order theory of an injective $\omega$-automatic structure (that is defined via Büchi-automata) of bounded degree. Here, injectivity means that every element of the structure is represented by a unique $\omega$-word from the underlying regular language.

In this paper, we achieve three goals:

\footnotetext{
* The second author acknowledges support from the DFG-project GELO.
} 
- We close the complexity gaps from [24] for string/tree automatic structures of bounded degree.

- We investigate, for the first time, the complexity of the uniform first-order theory (where the automatic presentation is part of the input) of string/tree automatic structures of bounded degree.

- We refine our complexity analysis using the growth function of a structure. This function measures the size of a sphere in the Gaifman graph depending on the radius of the sphere. The growth function of a structure of bounded degree can be at most exponential.

Our main results are the following:

- The uniform first-order theory for injective string automatic presentations is 2EXPSPACEcomplete. The lower bound already holds in the non-uniform setting, i.e. there exists a string automatic structure of bounded degree with a 2EXPSPACE-complete first-order theory.

- For every string automatic structure of bounded degree, where the growth function is polynomially bounded, the first-order theory is in EXPSPACE, and there exists an example with an EXPSPACE-complete first-order theory.

- The uniform first-order theory for injective tree automatic presentations belongs to 4EXPTIME; the non-uniform one to 3EXPTIME for arbitrary tree automatic structures, and to 2EXPTIME if the growth function is polynomial. Our bounds for the non-uniform problem are sharp, i.e., there are tree automatic structures of bounded degree (and polynomial growth) with a 3EXPTIME-complete (2EXPTIME-complete, resp.) first-order theory.

We conclude this paper with some results on the complexity of first-order fragments with fixed quantifier alternation depth one or two on string/tree automatic structures of bounded degree.

\section{Preliminaries}

Let $\Gamma$ be a finite alphabet and $w \in \Gamma^{*}$ be a finite word over $\Gamma$. The length of $w$ is denoted by $|w|$. We also write $\Gamma^{n}=\left\{w \in \Gamma^{*}|n=| w \mid\right\}$.

Let us define $\exp (0, x)=x$ and $\exp (n+1, x)=2^{\exp (n, x)}$ for $x \in \mathbb{N}$. We assume that the reader has some basic knowledge in complexity theory, see e.g. [26]. By Savitch's theorem, $\operatorname{NSPACE}(s(n)) \subseteq \operatorname{DSPACE}\left(s(n)^{2}\right)$ if $s(n) \geq \log (n)$. Hence, we can just write $\operatorname{SPACE}\left(s(n)^{O(1)}\right)$ for either $\operatorname{NSPACE}\left(s(n)^{O(1)}\right)$ or $\operatorname{DSPACE}\left(s(n)^{O(1)}\right)$. For $k \geq 1$, we denote with $k$ EXPSPACE (resp. $k$ EXPTIME) the class of all problems that can be accepted in space (resp. time) $\exp \left(k, n^{O(1)}\right)$ on a deterministic Turing machine. For 1EXPSPACE we write just EXPSPACE. A computational problem is called elementary if it belongs to $k$ EXPTIME for some $k \in \mathbb{N}$.

\subsection{Tree and string automata}

For our purpose it suffices to consider only tree automata on binary trees. Let $\Gamma$ be a finite alphabet. A finite binary tree over $\Gamma$ is a mapping $t: \operatorname{dom}(t) \rightarrow \Gamma$, where $\operatorname{dom}(t) \subseteq\{0,1\}^{*}$ is finite, nonempty, and satisfies the following closure condition for all $w \in\{0,1\}^{*}$ : if $\{w 0, w 1\} \cap$ $\operatorname{dom}(t) \neq \emptyset$, then also $w, w 0 \in \operatorname{dom}(t)$. With $T_{\Gamma}$ we denote the set of all finite binary trees over $\Gamma$. A (top-down) tree automaton over $\Gamma$ is a tuple $A=\left(Q, \Delta, q_{0}\right)$, where $Q$ is the finite set of states, $q_{0} \in Q$ is the initial state, and

$$
\Delta \subseteq(Q \times \Gamma \times Q \times Q) \cup(Q \times \Gamma \times Q) \cup(Q \times \Gamma)
$$


is the non-empty transition relation. A successful run of $A$ on a tree $t$ is a mapping $\rho$ : $\operatorname{dom}(t) \rightarrow Q$ such that (i) $\rho(\varepsilon)=q_{0}$ and (ii) for every $w \in \operatorname{dom}(t)$ with children $w 0, \ldots, w i$ (thus $-1 \leq i \leq 1$ ) we have $(\rho(w), t(w), \rho(w 0), \ldots, \rho(w i)) \in \Delta$. With $L(A)$ we denote the set of all finite binary trees $t$ such that there exists a successful run of $A$ on $t$. A set $L \subseteq T_{\Gamma}$ is called regular if there exists a finite tree automaton $A$ with $L=L(A)$.

A tree $t$ with $\operatorname{dom}(t) \subseteq 0^{*}$ can be considered as a nonempty string $t(\varepsilon) t(0) t(00) \ldots t\left(0^{n-1}\right)$ with $n=|\operatorname{dom}(t)|$. In the same spirit, a finite string automaton can be defined as a tree automaton, where the transition relation $\Delta$ in (1) satisfies $\Delta \subseteq(Q \times \Gamma \times Q) \cup(Q \times \Gamma)$.

We will need the following well known facts on string/tree automata: Emptiness (resp. inclusion) of the languages of string automata can be decided in nondeterministic logarithmic space (resp. polynomial space), whereas emptiness (resp. inclusion) of the languages of tree automata can be decided in polynomial time (resp. exponential time), see e.g. [8]. In all four cases completeness holds.

\subsection{Structures and first-order logic}

A signature is a finite set $\mathcal{S}$ of relational symbols, where every symbol $r \in \mathcal{S}$ has some fixed arity $m_{r}$. The notion of an $\mathcal{S}$-structure (or model) is defined as usual in logic. Note that we only consider relational structures. Sometimes, we will also use constants, but in our context, a constant $c$ can be always replaced by the unary relation $\{c\}$. Let us fix an $\mathcal{S}$-structure $\mathcal{A}=\left(A,\left(r^{\mathcal{A}}\right)_{r \in \mathcal{S}}\right)$, where $r^{\mathcal{A}} \subseteq A^{m_{r}}$. To simplify notation, we will write $a \in \mathcal{A}$ for $a \in A$. For $B \subseteq A$ we define the restriction $\mathcal{A}\left\lceil B=\left(B,\left(r^{\mathcal{A}} \cap B^{m_{r}}\right)_{r \in \mathcal{S}}\right)\right.$. Given further constants $a_{1}, \ldots, a_{n} \in \mathcal{A}$, we write $\left(\mathcal{A}, a_{1}, \ldots, a_{k}\right)$ for the structure $\left(A,\left(r^{\mathcal{A}}\right)_{r \in \mathcal{S}}, a_{1}, \ldots, a_{k}\right)$. In the rest of the paper, we will always identify a symbol $r \in \mathcal{S}$ with its interpretation $r^{\mathcal{A}}$.

A congruence on the structure $\mathcal{A}=\left(A,(r)_{r \in \mathcal{S}}\right)$ is an equivalence relation $\equiv$ on $A$ such that for every $r \in \mathcal{S}$ and all $a_{1}, b_{1}, \ldots, a_{m_{r}}, b_{m_{r}} \in A$ we have: If $\left(a_{1}, \ldots, a_{m_{r}}\right) \in r$ and $a_{1} \equiv b_{1}, \ldots, a_{m_{r}} \equiv b_{m_{r}}$, then also $\left(b_{1}, \ldots, b_{m_{r}}\right) \in r$. As usual, the equivalence class of $a \in A$ w.r.t. $\equiv$ is denoted by $[a]_{\equiv}$ or just $[a]$ and $A / \equiv$ denotes the set of all equivalence classes. We define the quotient structure $\mathcal{A} / \equiv=\left(A / \equiv,(r / \equiv)_{r \in \mathcal{S}}\right)$, where $r / \equiv=\left\{\left(\left[a_{1}\right], \ldots,\left[a_{m_{r}}\right]\right) \mid\right.$ $\left.\left(a_{1}, \ldots, a_{m_{r}}\right) \in r\right\}$.

The Gaifman-graph $G(\mathcal{A})$ of the $\mathcal{S}$-structure $\mathcal{A}$ is the following symmetric graph:

$$
G(\mathcal{A})=\left(A,\left\{(a, b) \in A \times A \mid \bigvee_{r \in \mathcal{S}} \exists\left(a_{1}, \ldots, a_{m_{r}}\right) \in r \exists j, k: a_{j}=a, a_{k}=b\right\}\right) .
$$

Thus, the set of nodes is the universe of $\mathcal{A}$ and there is an edge between two elements, if and only if they are contained in some tuple belonging to one of the relations of $\mathcal{A}$. With $d_{\mathcal{A}}(a, b)$, where $a, b \in \mathcal{A}$, we denote the distance between $a$ and $b$ in $G(\mathcal{A})$, i.e., it is the length of a shortest path connecting $a$ and $b$ in $G(\mathcal{A})$. For $a \in \mathcal{A}$ and $d \geq 0$ we denote with $S_{\mathcal{A}}(d, a)=\left\{b \in A \mid d_{\mathcal{A}}(a, b) \leq d\right\}$ the $d$-sphere around $a$. If $\mathcal{A}$ is clear from the context, then we will omit the subscript $\mathcal{A}$. We say that the structure $\mathcal{A}$ is locally finite if its Gaifman graph $G(\mathcal{A})$ is locally finite (i.e., every node has finitely many neighbors). Similarly, the structure $\mathcal{A}$ has bounded degree, if $G(\mathcal{A})$ has bounded degree, i.e., there exists a constant $\delta$ such that every $a \in A$ is adjacent to at most $\delta$ many other nodes in $G(\mathcal{A})$; the minimal such $\delta$ is called the degree of $\mathcal{A}$. For a structure $\mathcal{A}$ of bounded degree we can define its growth function as the mapping $g_{\mathcal{A}}: \mathbb{N} \rightarrow \mathbb{N}$ with $g_{\mathcal{A}}(n)=\max \left\{\left|S_{\mathcal{A}}(n, a)\right| \mid a \in \mathcal{A}\right\}$. Note that if the function $g_{\mathcal{A}}$ is not bounded then $g_{\mathcal{A}}(n) \geq n$ for all $n \geq 1$. For us, it is more convenient to not have a bounded function describing the growth. Therefore, we define the normalized 

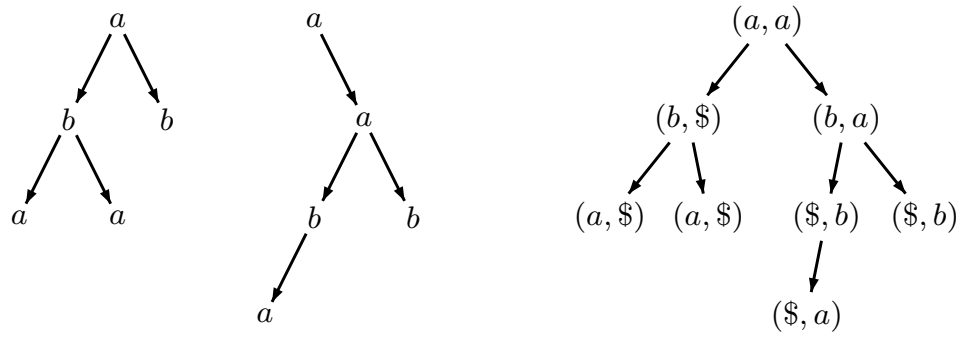

Fig. 1. The convolution of two trees

growth function $g_{\mathcal{A}}^{\prime}$ by $g_{\mathcal{A}}^{\prime}(n)=\max \left\{n, g_{\mathcal{A}}(n)\right\}$. Note that $g_{\mathcal{A}}$ and $g_{\mathcal{A}}^{\prime}$ are different only in the pathological case that all connected components of $\mathcal{A}$ contain at most $m$ elements (for some fixed $m$ ). Clearly, $g_{\mathcal{A}}^{\prime}(n)$ can grow at most exponentially. We say that $\mathcal{A}$ has exponential growth if $g_{\mathcal{A}}^{\prime}(n) \in 2^{\Omega(n)}$; if $g_{\mathcal{A}}^{\prime}(n) \in n^{O(1)}$, then $\mathcal{A}$ has polynomial growth.

To define logical formulas, we fix a countable infinite set $V$ of variables, which evaluate to elements of structures. Formulas over the signature $\mathcal{S}$ (or formulas if the the signature is clear from the context) are constructed from the atomic formulas $x=y$ and $r\left(x_{1}, \ldots, x_{m_{r}}\right)$, where $r \in \mathcal{S}$ and $x, y, x_{1}, \ldots, x_{m_{r}} \in V$, using the Boolean connectives $\vee$ and $\neg$ and existential quantification over variables from $V$. The Boolean connective $\wedge$ and universal quantification can be derived from these operators in the usual way. The quantifier depth of a formula $\varphi$ is the maximal nesting of quantifiers in $\varphi$. The notion of a free variable is defined as usual. A formula without free variables is called closed. If $\varphi\left(x_{1}, \ldots, x_{m}\right)$ is a formula with free variables among $x_{1}, \ldots, x_{m}$ and $a_{1}, \ldots, a_{m} \in \mathcal{A}$, then $\mathcal{A}=\varphi\left(a_{1}, \ldots, a_{m}\right)$ means that $\varphi$ evaluates to true in $\mathcal{A}$ when the free variable $x_{i}$ evaluates to $a_{i}$. The first-order theory of $\mathcal{A}$, denoted by $\operatorname{FOTh}(\mathcal{A})$, is the set of all closed formulas $\varphi$ such that $\mathcal{A} \models \varphi$.

\subsection{Structures from automata}

This section recalls string automatic and tree automatic structures and basic results about them. Details can be found in the survey [27].

Tree and string automatic structures String automatic structures were introduced in [14, their systematic study was later initiated by [16]. Tree automatic structures were introduced in [4], they generalize string automatic structures. Here, we will first introduce tree automatic structures. String automatic structures can be considered as a special case of tree automatic structures.

Let $\Gamma$ be a finite alphabet and let $\$ \notin \Gamma$ be an additional padding symbol. Let $t_{1}, \ldots, t_{m} \in$ $T_{\Gamma}$. We define the convolution $t=t_{1} \otimes \cdots \otimes t_{m}$, which is a finite binary tree over the alphabet $(\Gamma \cup\{\$\})^{m}$, as follows: $\operatorname{dom}(t)=\bigcup_{i=1}^{m} \operatorname{dom}\left(t_{i}\right)$ and for all $w \in \bigcup_{i=1}^{m} \operatorname{dom}\left(t_{i}\right)$ we define $t(w)=$ $\left(a_{1}, \ldots, a_{m}\right)$, where $a_{i}=t_{i}(w)$ if $w \in \operatorname{dom}\left(t_{i}\right)$ and $a_{i}=\$$ otherwise. In Fig. 1, the third tree is the convolution of the first two trees.

An $m$-dimensional (synchronous) tree automaton over $\Gamma$ is just a tree automaton $A$ over the alphabet $(\Gamma \cup\{\$\})^{m}$ such that $L(A) \subseteq\left\{t_{1} \otimes \cdots \otimes t_{n} \mid t_{1}, \ldots, t_{m} \in T_{\Gamma}\right\}$. Such an automaton defines an $m$-ary relation

$$
R(A)=\left\{\left(t_{1}, \ldots, t_{m}\right) \mid t_{1} \otimes \cdots \otimes t_{m} \in L(A)\right\} .
$$


A tree automatic presentation is a tuple $P=\left(\Gamma, A_{0}, A_{=},\left(A_{r}\right)_{r \in \mathcal{S}}\right)$, where:

$-\Gamma$ is a finite alphabet.

- $\mathcal{S}$ is a signature (the signature of $P$ ), as before $m_{r}$ is the arity of the symbol $r \in \mathcal{S}$.

- $A_{0}$ is a tree automaton over the alphabet $\Gamma$.

- For every $r \in \mathcal{S}, A_{r}$ is an $m_{r}$-dimensional tree automaton over the alphabet $\Gamma \cup\{\$\}$ such that $R\left(A_{r}\right) \subseteq L\left(A_{0}\right)^{m_{r}}$.

- $A_{=}$is a 2-dimensional tree automaton over the alphabet $\Gamma \cup\{\$\}$ such that $R\left(A_{=}\right) \subseteq$ $L\left(A_{0}\right) \times L\left(A_{0}\right)$ and $R\left(A_{=}\right)$is a congruence on the structure $\left(L\left(A_{0}\right),\left(R\left(A_{r}\right)\right)_{r \in \mathcal{S}}\right)$.

This presentation $P$ is called injective if $R\left(A_{=}\right)$is the identity relation on $L\left(A_{0}\right)$. In this case, we can omit the automaton $A_{=}$and identify $P$ with the tuple $\left(\Gamma, A_{0},\left(A_{r}\right)_{r \in \mathcal{S}}\right)$. The structure presented by $P$ is the quotient

$$
\mathcal{A}(P)=\left(L\left(A_{0}\right),\left(R\left(A_{r}\right)\right)_{r \in \mathcal{S}}\right) / R\left(A_{=}\right) \cdot
$$

A structure $\mathcal{A}$ is called tree automatic if there exists a tree automatic presentation $P$ such that $\mathcal{A} \simeq \mathcal{A}(P)$. We will write $[u]$ for the element $[u]_{R\left(A_{=}\right)}\left(u \in L\left(A_{0}\right)\right)$ of the structure $\mathcal{A}(P)$. We say that the presentation $P$ has bounded degree if the structure $\mathcal{A}(P)$ has bounded degree.

A string automatic presentation is a tree automatic presentation, where all tree automata are in fact string automata (as explained in Section 2.1), and a structure $\mathcal{A}$ is called string automatic if there exists a string automatic presentation $P$ such that $\mathcal{A} \simeq \mathcal{A}(P)$. Typical examples of string automatic structures are $(\mathbb{N},+)$ (Presburger's arithmetic), $(\mathbb{Q}, \leq)$, and all ordinals below $\omega^{\omega}$ [1610]. An example of a tree automatic structure, which is not string automatic is $(\mathbb{N}, \cdot)$ (the natural numbers with multiplication) [4], or the ordinal $\omega^{\omega}[10$. Examples of string automatic structures of bounded degree are transition graphs of Turing machines and Cayley-graphs of automatic groups [12] (or even right-cancellative monoids [29]).

Remark 2.1. Usually a tree automatic presentation for an $\mathcal{S}$-structure $\mathcal{A}=\left(A,(r)_{r \in \mathcal{S}}\right)$ is defined as a tuple $(\Gamma, L, h)$ such that

$-\Gamma$ is a finite alphabet,

$-L \subseteq T_{\Gamma}$ is a regular set of trees,

$-h: L \rightarrow A$ is a surjective function,

- the relation $\{(u, v) \in L \times L \mid h(u)=h(v)\}$ can be recognized by a 2-dimensional tree automaton, and

- for every $r \in \mathcal{S}$, the relation $\left\{\left(u_{1}, \ldots, u_{m_{r}}\right) \in L^{m_{r}} \mid\left(h\left(u_{1}\right), \ldots, h\left(u_{m_{r}}\right)\right) \in r\right\}$ can be recognized by an $m_{r}$-dimensional tree automaton.

Since for our considerations, tree automatic presentations are part of the input for algorithms, we prefer our definition, where a tree automatic presentation is a finite object (a tuple of finite tree automata), whereas in the standard definition, the presentation also contains the presentation map $h$. 
We will consider the following classes of tree automatic presentations:

$\mathrm{SA}=$ the class of all string automatic presentations

$\mathrm{SAb}=$ the class of all string automatic presentations of bounded degree

$\mathrm{iSA} b=$ the class of all injective string automatic presentations of bounded degree

$\mathrm{TA}=$ the class of all tree automatic presentations

$\mathrm{TAb}=$ the class of all tree automatic presentations of bounded degree

$\mathrm{iTAb}=$ the class of all injective tree automatic presentations of bounded degree

The model checking problem For the above classes of tree automatic presentations, we will be interested in the following decision problems.

Definition 2.2. Let $\mathrm{C}$ be a class of tree automatic presentations. Then the first-order model checking problem $\mathrm{FOMC}(\mathrm{C})$ for $\mathrm{C}$ denotes the set of all pairs $(P, \varphi)$ where $P \in \mathrm{C}$, and $\varphi$ is a closed formula over the signature of $P$ such that $\mathcal{A}(P) \models \varphi$.

If $\mathrm{C}=\{P\}$ is a singleton, then the model checking problem $\operatorname{FOMC}(\mathrm{C})$ for $\mathrm{C}$ can be identified with the first-order theory of the structure $\mathcal{A}(P)$. An algorithm deciding the model checking problem for a nontrivial class $\mathrm{C}$ decides the first-order theories of each element of $\mathrm{C}$ uniformly.

The following two results are the main motivations for investigating tree automatic structures.

Proposition 2.3 (cf. [16, 4]). There exists an algorithm that computes from a tree automatic presentation $P=\left(\Gamma, A_{0}, A_{=},\left(A_{r}\right)_{r \in \mathcal{S}}\right)$ and a formula $\varphi\left(x_{1}, \ldots, x_{m}\right)$ an $m$-dimensional tree automaton $A$ over $\Gamma$ with $R(A)=\left\{\left(u_{1}, \ldots, u_{m}\right) \in L\left(A_{0}\right)^{m} \mid \mathcal{A}(P) \models \varphi\left(\left[u_{1}\right], \ldots,\left[u_{m}\right]\right)\right\}$.

The automaton is constructed by induction on the structure of the formula $\varphi$ : disjunction corresponds to the disjoint union of automata, existential quantification to projection, and negation to complementation. The following result is a direct consequence.

Theorem 2.4 (cf. [16,4]). The model checking problem FOMC(TA) for all tree automatic presentations is decidable. In particular, the first-order theory $\operatorname{FOTh}(\mathcal{A})$ of every tree automatic structure $\mathcal{A}$ is decidable.

Remark 2.5. Strictly speaking, [164] device algorithms that, given a tree automatic presentation and a closed formula, decide whether the formula holds in the presented structure. But a priori, it is not clear whether it is decidable, whether a given tuple $\left(\Gamma, A_{0}, A_{=},\left(A_{r}\right)_{r \in \mathcal{S}}\right)$ is a tree automatic presentation. Lemma 2.8 below shows that TA is indeed decidable, which then completes the proof of this theorem.

Theorem 2.4 holds even if we add quantifiers for "there are infinitely many $x$ such that $\varphi(x)$ " [45] and "the number of elements satisfying $\varphi(x)$ is divisible by $k$ " (for $k \in \mathbb{N}$ ) [19]1. This implies in particular that it is decidable whether a tree automatic presentation describes a locally finite structure. But the decidability of the first-order theory is far from efficient,

\footnotetext{
${ }^{1}[19$ only provides the proofs for string automatic structures. These proofs are easily extended to tree automatic structures once the presentation is injective. But every tree automatic presentation can be transformed into an equivalent injective one [7, Cor. 4.2].
} 
since there are even string automatic structures with a nonelementary first-order theory [5]. For instance the structure $\left(\{0,1\}^{*}, s_{0}, s_{1}, \preceq\right)$, where $s_{i}=\left\{(w, w i) \mid w \in\{0,1\}^{*}\right\}$ for $i \in\{0,1\}$ and $\preceq$ is the prefix order on finite words, has a nonelementary first-order theory, see e.g. [9, Example 8.3]. A locally finite example (encoding the set of all finite labeled linearly ordered sets [25]) is as follows: the universe is the set $L=\left\{u \otimes v\left|u \in\{0,1\}^{+}, v \in 0^{*},\right| v|<| u \mid\right\}$. In addition, we have a partial order $\left\{\left(u \otimes v, u \otimes v^{\prime}\right) \in L \times L|| v|\leq| v^{\prime} \mid\right\}$ that encodes the union of all the linear order relations, and a unary relation $\{u \otimes v \in L \mid$ position $|v|$ in $u$ carries 1$\}$ that encodes the labeling.

First complexity results: the classes TA etc and boundedness This paper is concerned with the uniform and non-uniform complexity of the first-order theory of (some subclass of) tree automatic structures of bounded degree. Thus, we will consider algorithms that take as input tree automatic presentations (together with closed formulas). For complexity considerations, we have to define the size $|P|$ of a tree automatic presentation $P=\left(\Gamma, A_{0}, A_{=},\left(A_{r}\right)_{r \in \mathcal{S}}\right)$. First, let us define the size $|A|$ of an $m$-dimensional tree automaton $A=\left(Q, \Delta, q_{0}\right)$ over $\Gamma$. A transition tuple from $\Delta$ (see (11)) can be stored with at most $3 \log (|Q|)+m \log (|\Gamma|)$ many bits. Hence, up to constant factors, $\Delta$ can be stored in space $|\Delta| \cdot(\log (|Q|)+m \log (|\Gamma|))$. We can assume that every state is the first component of some transition tuple, i.e., $|Q| \leq|\Delta|$. Furthermore, the size of the basic alphabet $\Gamma$ can be bounded by $|\Delta|$ as well, but the dimension $m$ is independent from the size of $\Delta$. Since our complexity measures will be up to polynomial time reductions, it makes sense to define the size of the tree automaton $A$ to be $|A|=|\Delta| \cdot m$. We assume $\Delta$ to be nonempty, hence $|A| \geq 1$. The size of the presentation $P=\left(\Gamma, A_{0}, A_{=},\left(A_{r}\right)_{r \in \mathcal{S}}\right)$ is $|P|=\left|A_{0}\right|+\left|A_{=}\right|+\sum_{r \in \mathcal{S}}\left|A_{r}\right|$. Note that $|\mathcal{S}| \leq|P|$ and $m \leq|P|$, when $m$ is the maximal arity in $\mathcal{S}$.

It will be convenient to work with injective string (resp. tree) automatic presentations. The following lemma says that this is no restriction, at least if we do not consider complexity aspects.

Lemma 2.6 ([16, Cor. 4.3] and [7, Cor. 4.2]). From a given $P \in \mathrm{TA}$ we can compute effectively $P^{\prime} \in \mathrm{iTA}$ with $\mathcal{A}(P) \simeq \mathcal{A}\left(P^{\prime}\right)$. If $P \in \mathrm{SA}$, then $P^{\prime} \in \mathrm{iSA}$ with $\mathcal{A}(P) \simeq \mathcal{A}\left(P^{\prime}\right)$ can be computed in time $2^{O(|P|)}$.

Remark 2.7. In [7], only the existence of an equivalent injective tree automatic presentation is stated, but the proofs of [7, Prop. 3.1 and Theorem 4.1] are effective although the complexity is difficult to extract.

The following lemma shows that the classes of all tree and string automatic presentations are decidable and gives complexity bounds. While these two results are not surprising, it is not clear how to determine whether $\mathcal{A}(P)$ has bounded degree - this will be solved by Prop. 2.10 below.

Lemma 2.8. The class TA is in EXPTIME, and the class SA belongs to PSPACE.

Proof. We start with a proof of the first statement. Suppose we are given a finite alphabet $\Gamma$, tree automata $A_{0}$ over $\Gamma$, and multi-dimensional tree automata $A_{=}$and $A_{r}$ for $r \in \mathcal{S}$ over $\Gamma \cup$ $\{\$\}$. In a first step, we check that $L\left(A_{=}\right)$and $L\left(A_{r}\right)$ are languages of convolutions of elements of $L\left(A_{0}\right)$, in particular

$$
L\left(A_{r}\right) \subseteq \underbrace{L\left(A_{0}\right) \otimes L\left(A_{0}\right) \cdots \otimes L\left(A_{0}\right)}_{m_{r} \text { times }}
$$


where $m_{r}$ is the arity of the automaton $A_{r}$. An automaton for the right-hand side has size $\left|A_{0}\right|^{m_{r}}$. Thus, the inclusion can be decided in time exponential in $\left|A_{r}\right|+\left|A_{0}\right|^{m_{r}}$. Since $m_{r}$ depends on the input, this yields a doubly exponential algorithm. Alternatively, we proceed as follows:

(a) We check that no tree from $L\left(A_{r}\right)$ contains the label $(\$, \ldots, \$)$. To this aim, replace in all transitions of $A_{r}$ the letters from $(\Gamma \cup\{\$\})^{m_{r}} \backslash\{(\$, \ldots, \$)\}$ by $\top$ and the letter $(\$, \ldots, \$)$ by $\perp$ and check whether the language of the resulting automaton $A_{r}^{\prime}$ is contained in $T_{\{\top\}}$ (the set of all $T$-labeled binary trees). Since the set $T_{\{T\}}$ can be accepted by a fixed automaton, this inclusion can be decided in polynomial time.

(b) Let $H \subseteq T_{\Gamma \cup\{\$\}}$ denote the set of those trees $t$ whose $\Gamma$-labeled nodes form an initial segment of $t$ that belongs to $L\left(A_{0}\right)$. To accept $H$, we extend $A_{0}$ as follows (where $a \in \Gamma$ ): - We add a new state $q_{\$}$ and transitions $\left(q_{\$}, \$\right),\left(q_{\$}, \$, q_{\$}\right)$, and $\left(q_{\$}, \$, q_{\$}, q_{\$}\right)$.

- For each transition $(p, a, q)$, we add the transition $\left(p, a, q, q_{\$}\right)$.

- For each transition $(p, a)$, we add the transitions $\left(p, a, q_{\$}\right)$ and $\left(p, a, q_{\$}, q_{\$}\right)$.

Let $A_{0}^{\$}$ denote the resulting tree automaton and, for $1 \leq i \leq m_{r}$, let $A_{r}^{i}$ denote the projection of $A_{r}$ to its $i^{\text {th }}$ component. Then we check, for all $1 \leq i \leq m_{r}$ whether $L\left(A_{r}^{i}\right) \subseteq L\left(A_{0}^{\$}\right)$ which can be done in exponential time.

All these tests are passed if and only if (2) holds for $A_{r}$. In particular, we can from now on speak of the relations $R\left(A_{=}\right)$and $R\left(A_{r}\right)$ over $L\left(A_{0}\right)$.

It remains to be checked that $R\left(A_{=}\right)$is a congruence on the structure $\left(L\left(A_{0}\right),\left(R\left(A_{r}\right)\right)_{r \in \mathcal{S}}\right)$. For this, we proceed as follows

(c) First build 2-dimensional tree automata $A_{\circ}, A_{-1}$, and $A_{\text {id }}$ of polynomial size with $R\left(A_{\circ}\right)=$ $R\left(A_{=}\right) \circ R\left(A_{=}\right), R\left(A_{-1}\right)=R\left(A_{=}\right)^{-1}$, and $R\left(A_{\text {id }}\right)=\left\{(t, t) \mid t \in L\left(A_{0}\right)\right\}$. Then check $R\left(A_{\circ}\right) \cup R\left(A_{-1}\right) \cup R\left(A_{\text {id }}\right) \subseteq R\left(A_{=}\right)$which can be done in exponential time. This test is passed if and only if $R\left(A_{=}\right)$is an equivalence relation on $L\left(A_{0}\right)$.

(d) For each $r \in \mathcal{S}$, first construct an $2 m_{r}$-dimensional tree automaton $A_{r}^{\prime}$ such that the tuple $\left(s_{1}, \ldots, s_{m_{r}}, t_{1}, \ldots, t_{m_{r}}\right)$ belongs to $R\left(A_{r}^{\prime}\right)$ if and only if $\left(s_{i}, t_{i}\right) \in R\left(A_{=}\right)$for all $1 \leq i \leq m_{r}$ and $\left(t_{1}, \ldots, t_{m_{r}}\right) \in R\left(A_{r}\right)$. This can be achieved by running $m_{r}$ copies of $A_{=}$as well as one copy of $A_{r}$ in parallel. Then project the automaton $A_{r}^{\prime}$ onto the first $m_{r}$ components and check whether the relation accepted by the resulting tree automaton is contained in $R\left(A_{r}\right)$. Although $A_{r}^{\prime}$ has exponential size (since $m_{r}$ depends on the presentation $P$ ), this can be done again in exponential time: we complement $A_{r}$, take the intersection with $A_{r}^{\prime}$ and check the resulting automaton (of exponential size) for emptiness.

This finishes the proof of the first statement. To prove the second, one can proceed analogously using that the inclusion problem for string automata belongs to PSPACE.

From the lower bounds for inclusion of string/tree automata, it follows easily that the upper bounds in Lemma 2.8 are sharp.

The following lemma says that the Gaifman graph of a string (resp. tree) automatic structure is effectively string (resp. tree) automatic. This is an immediate consequence of Prop. 2.3. so the novelty lies in the estimation of the complexity.

Lemma 2.9. From a given tree (string) automatic presentation $P=\left(\Gamma, A_{0}, A_{=},\left(A_{r}\right)_{r \in \mathcal{S}}\right)$ one can construct a 2-dimensional tree (string) automaton A such that

$$
R(A)=\left\{(u, v) \in L\left(A_{0}\right) \times L\left(A_{0}\right) \mid([u],[v]) \text { is an edge of the Gaifman-graph } G(\mathcal{A}(P))\right\} .
$$

If $m$ is the maximal arity in $\mathcal{S}$, then $A$ can be computed in time $O\left(m^{2} \cdot|P|^{2}\right) \leq|P|^{O(1)}$. 
Proof. We only give the proof for string automatic presentations, the tree automatic case can be shown verbatim. Let $E$ be the edge relation of the Gaifman-graph $G(\mathcal{A}(P))$. Note that for all $u, v \in L\left(A_{0}\right)$ we have $([u],[v]) \in E$ iff for some $r \in \mathcal{S}$ of arity $m_{r} \leq m$ and $1 \leq i, j \leq m_{r}$, there exist $u_{1}, \ldots, u_{m_{r}} \in L\left(A_{0}\right)$ with $\left(u_{1}, \ldots, u_{m_{r}}\right) \in R\left(A_{r}\right), u=u_{i}$, and $v=u_{j}$. Let $r \in \mathcal{S}$ and $1 \leq i, j \leq m_{r}$. Projecting the automaton $A_{r}$ onto the tracks $i$ and $j$, one obtains a 2-dimensional automaton accepting all pairs $(u, v) \in \Gamma^{*} \times \Gamma^{*}$ such that there exists $\left(u_{1}, \ldots, u_{m_{r}}\right) \in R\left(A_{r}\right)$ with $u=u_{i}$ and $v=u_{j}$. Then the disjoint union of all these automata (for $r \in \mathcal{S}$ and $1 \leq i, j \leq m_{r}$ ) satisfies (3). Since $|\mathcal{S}| \leq|P|$, the construction can be performed in time $O\left(m^{2} \cdot|P|^{2}\right)$.

Lemma 2.9 allows to show that also the bounded classes TAb etc. are decidable:

Proposition 2.10. The following hold:

(a) The class TAb is decidable.

(b) The class iTAb can be decided in exponential time (in fact, it can be checked in polynomial time whether a given $P \in \mathrm{iTA}$ has bounded degree).

(c) The class SAb can be decided in exponential time.

Proof. For statement (a), let $P \in \mathrm{TA}$ (which is decidable by Lemma 2.8 in exponential time). By Lemma 2.6, we can assume $P$ to be injective. By Lemma 2.9 we can compute an automaton $A$ with (3) , i.e., $A$ defines the edge relation of the Gaifman-graph of $\mathcal{A}(P)$. Since $P$ was assumed to be injective (i.e. every equivalence class $[u]$ is the singleton $\{u\}), \mathcal{A}(P)$ is of bounded degree iff $A$ (seen as a transducer) is finite-valued. But this is decidable in polynomial time 3028 . This finishes the proof of (a).

Next consider statement (b): Provided the input is guaranteed to be an injective tree automatic presentation, the polynomial time bound follows from the arguments above since there is no need to apply Lemma 2.6. It remains to decide whether the input is indeed an injective tree automatic presentation: Using Lemma 2.8, it suffices to decide injectivity which can be done in exponential time by checking inclusion of $L\left(A_{=}\right)$in the convolution of the identity on $T_{\Gamma}$.

For (c), where we start with a string automatic presentation (which can be decided in polynomial space and therefore exponential time by Lemma 2.8), the initial application of Lemma 2.6 leads to an exponential blow-up, which gives in total an exponential running time for deciding the class SAb.

Finally, since we deal with structures of bounded degree, it will be important to estimate the degree of such a structures given its presentation. Such estimates are provided by the following result.

Proposition 2.11. The following hold:

(a) If $P \in \mathrm{iSAb}$, then the degree of the structure $\mathcal{A}(P)$ is bounded by $\exp \left(1,|P|^{O(1)}\right)$.

(b) If $P \in \mathrm{iTAb}$, then the degree of the structure $\mathcal{A}(P)$ is bounded by $\exp \left(2,|P|^{O(1)}\right)$.

(c) If $P \in \mathrm{SAb}$, then the degree of the structure $\mathcal{A}(P)$ is bounded by $\exp \left(2,|P|^{O(1)}\right)$.

Proof. For statement (a) let $P \in$ iSAb. From Lemma 2.9, we can construct a string automaton $A$ of size $|P|^{O(1)}$ that accepts the edge relation of the Gaifman graph of $\mathcal{A}(P)$. Then the degree of $\mathcal{A}(P)$ equals the maximal outdegree of the relation $R(A)$. For string transducer, this number is exponential in the size of $A$, i.e., it is in $\exp \left(1,|P|^{O(1)}\right)[30]$. 
For (b) we can use a similar argument. But since the maximal outdegree of the relation recognized by a tree transducer $A$ is doubly exponential in the size of $A$ [28], we obtain the bound $\exp \left(2,|P|^{O(1)}\right)$ for the degree of $\mathcal{A}(P)$.

Finally statement (c) follows immediately from Lemma 2.6 and (a).

The bounds on injective string (resp. tree) automatic presentations in Prop. 2.11 are sharp: Let $E_{n}=\left\{(u w, v w)\left|u, v, w, \in\{a, b\}^{*},\right| u|=| v \mid=n\right\}$. Then the structure $\left(\{a, b\}^{*}, E_{n}\right)$ has an injective string automatic presentation of size $O(n)$. The degree of this structure is $2^{n}$. Similarly, let $E_{n}^{\prime}$ the set of all pairs $\left(t_{1}, t_{2}\right) \in T_{\{a, b\}} \times T_{\{a, b\}}$ of trees that differ at most in the first $n$ levels. Then $\left(T_{\{a, b\}}, E_{n}^{\prime}\right)$ allows an injective tree automatic presentation of size $O(n)$ and the degree of this structure is doubly exponential in $n$. But it is not clear whether the doubly exponential bound for automatic presentations in Prop. 2.11(c) can be realized. Moreover, we cannot give any bound for general tree automatic presentations since, as already remarked, [7] does not provide any estimate on the size of an equivalent injective tree automatic presentation.

\section{Upper bounds}

It is the aim of this section to give an algorithm that decides the theory of a string/tree automatic structure of bounded degree. The algorithm from Theorem 2.4 (that in particular solves this problem) is based on Prop. 2.3, i.e., the inductive construction of an automaton accepting all satisfying assignments. Differently, we base our algorithm on Gaifman's Theorem 3.1, i.e., on the combinatorics of spheres. We therefore start with some model theory.

\subsection{Model-theoretic background}

The following locality principle of Gaifman implies that super-exponential distances cannot be handled in first-order logic:

Theorem 3.1 ([13]). Let $\mathcal{A}$ be a structure, $\left(a_{1}, \ldots, a_{k}\right),\left(b_{1}, \ldots, b_{k}\right) \in \mathcal{A}^{k}, d \geq 0$, and $D_{1}, \ldots, D_{k} \geq 2^{d}$ such that

$$
\left(\mathcal{A} \uparrow\left(\bigcup_{i=1}^{k} S\left(D_{i}, a_{i}\right)\right), a_{1}, \ldots, a_{k}\right) \simeq\left(\mathcal{A} \uparrow\left(\bigcup_{i=1}^{k} S\left(D_{i}, b_{i}\right)\right), b_{1}, \ldots, b_{k}\right)
$$

Then, for every formula $\varphi\left(x_{1}, \ldots, x_{k}\right)$ of quantifier depth at most $d$, we have:

$$
\mathcal{A}=\varphi\left(a_{1}, \ldots, a_{k}\right) \Longleftrightarrow \mathcal{A} \models \varphi\left(b_{1}, \ldots, b_{k}\right) \text {. }
$$

Note that (41) says that there is an isomorphism between the two induced substructures $\mathcal{A} \uparrow\left(\bigcup_{i=1}^{k} S\left(D_{i}, a_{i}\right)\right)$ and $\mathcal{A} \uparrow\left(\bigcup_{i=1}^{k} S\left(D_{i}, b_{i}\right)\right)$ that maps $a_{i}$ to $b_{i}$ for all $1 \leq i \leq k$.

Let $\mathcal{S}$ be a signature and let $k, d \in \mathbb{N}$ with $0 \leq k \leq d$. A potential $(d, k)$-sphere is a tuple $\left(\mathcal{B}, b_{1}, \ldots, b_{k}\right)$ such that the following holds:

$-\mathcal{B}$ is an $\mathcal{S}$-structure with $b_{1}, \ldots, b_{k} \in \mathcal{B}$.

- For all $b \in \mathcal{B}$ there exists $1 \leq i \leq k$ such that $d_{\mathcal{B}}\left(b_{i}, b\right) \leq 2^{d-i}$. 
There is only one $(d, 0)$-sphere namely the empty sphere $\emptyset$. For our later applications, $\mathcal{B}$ will be always a finite structure, but in this subsection finiteness is not needed. The potential $(d, k)$-sphere $\left(\mathcal{B}, b_{1}, \ldots, b_{k}\right)$ is realizable in the structure $\mathcal{A}$ if there exist $a_{1}, \ldots, a_{k} \in \mathcal{A}$ such that

$$
\left(\mathcal{A} \uparrow\left(\bigcup_{i=1}^{k} S\left(2^{d-i}, a_{i}\right)\right), a_{1}, \ldots, a_{k}\right) \simeq\left(\mathcal{B}, b_{1}, \ldots, b_{k}\right) .
$$

Let $\sigma=\left(\mathcal{B}, b_{1}, \ldots, b_{k}\right)$ be a potential $(d, k)$-sphere and let $\sigma^{\prime}=\left(\mathcal{B}^{\prime}, b_{1}^{\prime}, \ldots, b_{k}^{\prime}, b_{k+1}^{\prime}\right)$ be a potential $(d, k+1)$-sphere $(k+1 \leq d)$. Then $\sigma^{\prime}$ extends $\sigma$ (abbreviated $\left.\sigma \preceq \sigma^{\prime}\right)$ if

$$
\left(\mathcal{B}^{\prime} \uparrow\left(\bigcup_{i=1}^{k} S\left(2^{d-i}, b_{i}\right)\right), b_{1}^{\prime}, \ldots, b_{k}^{\prime}\right) \simeq\left(\mathcal{B}, b_{1}, \ldots, b_{k}\right) .
$$

The following definition is the basis for our decision procedure.

Definition 3.2. Let $\mathcal{A}$ be an $\mathcal{S}$-structure, $\psi\left(y_{1}, \ldots, y_{k}\right)$ a formula of quantifier depth at most $d$, and let $\sigma=\left(\mathcal{B}, b_{1}, \ldots, b_{k}\right)$ be a potential $(d+k, k)$-sphere. The Boolean value $\psi_{\sigma} \in$ $\{0,1\}$ is defined inductively as follows:

- If $\psi\left(y_{1}, \ldots, y_{k}\right)$ is an atomic formula, then

$$
\psi_{\sigma}= \begin{cases}0 & \text { if } \mathcal{B} \models \psi\left(b_{1}, \ldots, b_{k}\right) \\ 1 & \text { if } \mathcal{B} \not \forall \psi\left(b_{1}, \ldots, b_{k}\right) .\end{cases}
$$

- If $\psi=\neg \theta$, then $\psi_{\sigma}=1-\theta_{\sigma}$.

- If $\psi=\alpha \vee \beta$, then $\psi_{\sigma}=\max \left(\alpha_{\sigma}, \beta_{\sigma}\right)$.

- If $\psi\left(y_{1}, \ldots, y_{k}\right)=\exists y_{k+1} \theta\left(y_{1}, \ldots, y_{k}, y_{k+1}\right)$ then

$$
\psi_{\sigma}=\max \left\{\theta_{\sigma^{\prime}} \mid \sigma^{\prime} \text { is a realizable potential }(d+k, k+1) \text {-sphere with } \sigma \preceq \sigma^{\prime}\right\} \text {. }
$$

The following result ensures for every closed formula $\psi$ that $\psi_{\emptyset}=1$ if and only if $\mathcal{A}=\psi$. Hence the above definition can possibly be used to decide validity of the formula $\varphi$ in the structure $\mathcal{A}$.

Proposition 3.3. Let $\mathcal{S}$ be a signature, $\mathcal{A}$ an $\mathcal{S}$-structure with $a_{1}, \ldots, a_{k} \in \mathcal{A}, \psi\left(y_{1}, \ldots, y_{k}\right)$ a formula of quantifier depth at most $d$, and $\sigma=\left(\mathcal{B}, b_{1}, \ldots, b_{k}\right)$ a potential $(d+k, k)$-sphere with

$$
\left(\mathcal{A} \uparrow\left(\bigcup_{i=1}^{k} S\left(2^{d+k-i}, a_{i}\right)\right), a_{1}, \ldots, a_{k}\right) \simeq\left(\mathcal{B}, b_{1}, \ldots, b_{k}\right) .
$$

Then $\mathcal{A}=\psi\left(a_{1}, \ldots, a_{k}\right) \Longleftrightarrow \psi_{\sigma}=1$.

Proof. We prove the lemma by induction on the structure of the formula $\psi$. First assume that $\psi$ is atomic, i.e. $d=0$. Then we have:

$$
\begin{aligned}
\psi_{\sigma}=1 & \stackrel{\text { (5) }}{\Longleftrightarrow} \mathcal{B}=\psi\left(b_{1}, \ldots, b_{k}\right) \\
& \stackrel{(7)}{\Longleftrightarrow} \mathcal{A} \uparrow\left(\bigcup_{i=1}^{k} S\left(2^{k-i}, a_{i}\right)\right) \models \psi\left(a_{1}, \ldots, a_{k}\right) \\
& \Longleftrightarrow \mathcal{A}=\psi\left(a_{1}, \ldots, a_{k}\right),
\end{aligned}
$$


where the last equivalence holds since $\psi$ is atomic.

The cases $\psi=\neg \theta$ and $\psi=\alpha \vee \beta$ are straightforward and therefore omitted.

We finally consider the case $\psi\left(y_{1}, \ldots, y_{k}\right)=\exists y_{k+1} \theta\left(y_{1}, \ldots, y_{k}, y_{k+1}\right)$.

First assume that $\psi_{\sigma}=1$. By (6) $)$, there exists a realizable potential $(d+k, k+1)$-sphere $\sigma^{\prime}$ with $\sigma \preceq \sigma^{\prime}$ and $\theta_{\sigma^{\prime}}=1$. Since $\sigma^{\prime}$ is realizable, there exist $a_{1}^{\prime}, \ldots, a_{k}^{\prime}, a_{k+1}^{\prime} \in \mathcal{A}$ with

$$
\left(\mathcal{A} \uparrow\left(\bigcup_{i=1}^{k+1} S\left(2^{d+k-i}, a_{i}^{\prime}\right)\right), a_{1}^{\prime}, \ldots, a_{k}^{\prime}, a_{k+1}^{\prime}\right) \simeq\left(\mathcal{B}^{\prime}, b_{1}, \ldots, b_{k}, b_{k+1}\right)=\sigma^{\prime} .
$$

By induction, we have $\mathcal{A} \models \theta\left(a_{1}^{\prime}, \ldots, a_{k}^{\prime}, a_{k+1}^{\prime}\right)$ and therefore $\mathcal{A} \models \psi\left(a_{1}^{\prime}, \ldots, a_{k}^{\prime}\right)$. From (7), (8), and $\sigma \preceq \sigma^{\prime}$, we also obtain

$$
\left(\mathcal{A} \uparrow\left(\bigcup_{i=1}^{k} S\left(2^{d+k-i}, a_{i}^{\prime}\right)\right), a_{1}^{\prime}, \ldots, a_{k}^{\prime}\right) \simeq\left(\mathcal{A} \uparrow\left(\bigcup_{i=1}^{k} S\left(2^{d+k-i}, a_{i}\right)\right), a_{1}, \ldots, a_{k}\right)
$$

and therefore by Gaifman's Theorem $3.1 \mathcal{A}=\psi\left(a_{1}, \ldots, a_{k}\right)$.

Conversely, let $a_{k+1} \in \mathcal{A}$ with $\mathcal{A} \models \theta\left(a_{1}, \ldots, a_{k}, a_{k+1}\right)$. Let $\sigma^{\prime}=\left(\mathcal{B}^{\prime}, b_{1}, \ldots, b_{k}, b_{k+1}\right)$ be the unique (up to isomorphism) potential $(d+k, k+1)$-sphere such that

$$
\left(\mathcal{A} \uparrow\left(\bigcup_{i=1}^{k+1} S\left(2^{d+k-i}, a_{i}\right)\right), a_{1}, \ldots, a_{k}, a_{k+1}\right) \simeq\left(\mathcal{B}^{\prime}, b_{1}, \ldots, b_{k}, b_{k+1}\right) .
$$

Then (7) implies $\sigma \preceq \sigma^{\prime}$. Moreover, by (9), $\sigma^{\prime}$ is realizable in $\mathcal{A}$, and $\mathcal{A} \models \theta\left(a_{1}, \ldots, a_{k}, a_{k+1}\right)$ implies by induction $\theta_{\sigma^{\prime}}=1$. Hence, by (6), we get $\psi_{\sigma}=1$ which finishes the proof of the lemma.

\subsection{The decision procedure}

Now suppose we want to decide whether the closed formula $\varphi$ holds in a tree automatic structure $\mathcal{A}$ of bounded degree. By Prop. 3.3 it suffices to compute the Boolean value $\varphi_{\emptyset}$. This computation will follow the inductive definition of $\varphi_{\sigma}$ from Def. 3.2. Since every $(d, k)$-sphere that is realizable in $\mathcal{A}$ is finite, we only have to deal with finite spheres. The crucial part of our algorithm is to determine whether a finite potential $(d, k)$-sphere is realizable in $\mathcal{A}$. In the following, for a finite potential $(d, k)$-sphere $\sigma=\left(\mathcal{B}, b_{1}, \ldots, b_{k}\right)$, we denote with $|\sigma|$ the number of elements of $\mathcal{B}$ and with $\delta(\sigma)$ we denote the degree of the finite structure $\mathcal{B}$. We have to solve the following realizability problem:

Definition 3.4. Let $\mathrm{C}$ be a class of tree automatic presentations. Then the realizability problem $\operatorname{REAL}(\mathrm{C})$ for $\mathrm{C}$ denotes the set of all pairs $(P, \sigma)$ where $P \in \mathrm{C}$ and $\sigma$ is a finite potential $(d, k)$-sphere over the signature of $P$ for some $0 \leq k \leq d$ such that $\sigma$ can be realized in $\mathcal{A}(P)$.

Lemma 3.5. The problems $\mathrm{REAL}(\mathrm{iSA})$ and $\mathrm{REAL}(\mathrm{iTA})$ are decidable. More precisely:

- Let $P \in \mathrm{iSA}$ and let $m$ be the maximal arity of a relation in $\mathcal{A}(P)$. Let $\sigma$ be a finite potential $(d, k)$-sphere over the signature of $P$. Then it can be checked in space $|\sigma|^{O(m)} \cdot|P|^{2} \cdot 2^{O(\delta(\sigma))}$, whether $\sigma$ is realizable in $\mathcal{A}(P)$.

- If $P \in \mathrm{iTA}$, then realizability can be checked in time $\exp \left(1,|\sigma|^{O(m)} \cdot|P|^{2} \cdot 2^{O(\delta(\sigma))}\right)$. 
Proof. We first prove the statement on injective string automatic presentations. Let $P=$ $\left(\Gamma, A_{0},\left(A_{r}\right)_{r \in \mathcal{S}}\right) \in \mathrm{iSA}$. Let $\sigma=\left(\mathcal{B}, b_{1}, \ldots, b_{k}\right)$ and let $c_{1}, \ldots, c_{|\sigma|}$ be a list of all elements of $\mathcal{B}$. Note that every $b_{i}$ occurs in this list. Let $E_{\mathcal{A}(P)}$ be the edge relation of the Gaifman graph $G(\mathcal{A}(P))$ and $E_{\mathcal{B}}$ that of the Gaifman graph $G(\mathcal{B})$. Then $\sigma$ is realizable in $\mathcal{A}(P)$ if and only if there are words $u_{1}, \ldots, u_{|\sigma|} \in \Gamma^{*}$ such that

(a) $u_{i} \in L\left(A_{0}\right)$ for all $1 \leq i \leq|\sigma|$,

(b) $u_{i} \neq u_{j}$ for all $1 \leq i<j \leq|\sigma|$,

(c) $\left(u_{i_{1}}, \ldots, u_{i_{m_{r}}}\right) \in R\left(A_{r}\right)$ for all $r \in \mathcal{S}$ and all $\left(c_{i_{1}}, \ldots, c_{i_{m_{r}}}\right) \in r^{\mathcal{B}}$,

(d) $\left(u_{i_{1}}, \ldots, u_{i_{m_{r}}}\right) \notin R\left(A_{r}\right)$ for all $r \in \mathcal{S}$ and all $\left(c_{i_{1}}, \ldots, c_{i_{m_{r}}}\right) \in \mathcal{B}^{m_{r}} \backslash r^{\mathcal{B}}$, and

(e) there is no $v \in L\left(A_{0}\right)$ such that, for some $1 \leq j \leq|\sigma|$ and $1 \leq i \leq k$ with $d\left(c_{j}, b_{i}\right)<2^{d-i}$, we have

(e.1) $\left(u_{j}, v\right) \in E_{\mathcal{A}(P)}$ and

(e.2) $v \notin\left\{u_{p} \mid\left(c_{j}, c_{p}\right) \in E_{\mathcal{B}}\right\}$.

Then (a-d) express that the mapping $c_{i} \mapsto u_{i}$ is well-defined and an embedding of $\mathcal{B}$ into $\mathcal{A}(P)$. In (e), $\left(u_{j}, v\right) \in E_{\mathcal{A}(P)}$ implies that $v$ belongs to $\bigcup_{1 \leq i \leq k} S\left(2^{d-i}, u_{i}\right)$. Hence (e) expresses that all elements of $\bigcup_{1 \leq i \leq k} S\left(2^{d-i}, u_{i}\right)$ belong to the image of this embedding.

We now construct a $|\sigma|$-dimensional automaton $A$ over the alphabet $\Gamma$ that checks (a-e). At the end, we have to check the language of this automaton for non-emptiness. The automaton $A$ is the direct product of automata $A_{a}, A_{b}, A_{c}, A_{d}$, and $A_{e}$ that check the conditions separately. Then $A_{a}$ is the direct product of $|\sigma|$ many copies of the automaton $A_{0}$, hence $A_{a}$ has at most $|P|^{|\sigma|}$ many states.

Next, the automaton for (b) is the direct product of $O\left(|\sigma|^{2}\right)$ many copies of an automaton of fixed size (that checks whether two tracks are different). Hence, this automaton has $2^{|\sigma|^{O(1)}}$ many states.

The automaton $A_{c}$ is again a direct product, this time of one automaton for each $r \in \mathcal{S}$ (and therefore of at most $|P|$ many automata). Each of these automata is the direct product of $\left|r^{\mathcal{B}}\right|$ many copies of the automaton $A_{r}$. Since the arity of $r \in \mathcal{S}$ is bounded by $m$, we have $\left|r^{\mathcal{B}}\right| \leq|\sigma|^{m}$. Hence, the automaton $A_{c}$ has at most $\left(|P|^{|\sigma|^{m}}\right)^{|P|}=|P|^{|P| \cdot|\sigma|^{m}}$ many states. For $A_{d}$, we can argue similarly, but this time using copies of the complement of the automaton $A_{r}$. This yields for $A_{d}$ the bound $\left(2^{|P|}\right)^{|P| \cdot|\sigma|^{m}}=\exp \left(1,|P|^{2} \cdot|\sigma|^{m}\right)$ on the number of states.

It remains to construct the automaton $A_{e}$. For this, we first construct its complement, i.e., an automaton $A_{e}^{\prime}$ that checks for the existence of $v \in L\left(A_{0}\right)$ with the desired properties. This automaton $A_{e}^{\prime}$ is the disjoint union of at most $|\sigma|$ many automata, one for each $1 \leq j \leq|\sigma|$ such that there exists $1 \leq i \leq k$ with $d\left(c_{j}, b_{i}\right)<2^{d-i}$. Any of these components consists of the direct product of automata $A_{e .1}$ and $A_{e .2}$ checking (e.1) and (e.2), respectively. By Lemma 2.9, $A_{e .1}$ hast at most $m^{2} \cdot|P|^{2}$ many states. Recall that the degree of $\mathcal{B}$ is $\delta(\sigma)$. Hence, the set $\left\{u_{p} \mid\left(c_{j}, c_{p}\right) \in E_{\mathcal{B}}\right\}$ contains at most $\delta(\sigma)$ many elements. Thus, (e.2) can be checked by an automaton $A_{e .2}$ with $2^{O(\delta(\sigma))}$ many states. Hence, $A_{e}^{\prime}$ is the disjoint union of at most $|\sigma|$ copies of an automaton of size $|P|^{2} \cdot m^{2} \cdot 2^{O(\delta(\sigma))}$ and therefore has at most $|\sigma| \cdot|P|^{2} \cdot m^{2} \cdot 2^{O(\delta(\sigma))}$ many states. Now the number of states of $A_{e}$ can be bound by $\exp \left(1,|\sigma| \cdot|P|^{2} \cdot m^{2} \cdot 2^{O(\delta(\sigma))}\right)$.

In summary, the automaton $A$ has at most

$$
|P|^{|\sigma|} \cdot 2^{|\sigma|^{O(1)}} \cdot|P|^{|P| \cdot|\sigma|^{m}} \cdot 2^{|P|^{2} \cdot|\sigma|^{m}} \cdot 2^{|\sigma| \cdot|P|^{2} \cdot m^{2} \cdot 2^{O(\delta(\sigma))}} \leq \exp \left(1,|\sigma|^{O(m)} \cdot|P|^{2} \cdot 2^{O(\delta(\sigma))}\right)
$$


many states. Hence checking emptiness of its language (and therefore realizability of $\sigma$ in $\mathcal{A}(P)$ ) can be done in space logarithmic to the number of states, i.e., in space $|\sigma|^{O(m)} \cdot|P|^{2}$. $2^{O(\delta(\sigma))}$ which proves the statement for string automatic presentations.

For injective tree automatic presentations, the construction and size estimate for $A$ are the same as above. But emptiness of tree automata can only be checked in deterministic polynomial time (and not in logspace unless $\mathrm{NL}=\mathrm{P}$ ). Hence, emptiness of $A$ can be checked in time $\exp \left(1,|\sigma|^{O(m)} \cdot|P|^{2} \cdot 2^{O(\delta(\sigma))}\right)$.

In the following, for a tree automatic presentation $P$ of bounded degree, we denote with $g_{P}^{\prime}=g_{\mathcal{A}(P)}^{\prime}$ the normalized growth function of the structure $\mathcal{A}(P)$.

Theorem 3.6. The model checking problem $\mathrm{FOMC}(\mathrm{TAb})$ is decidable, i.e., on input of a tree automatic presentation $P$ of bounded degree and a closed formula $\varphi$ over the signature of $P$, one can effectively determine whether $\mathcal{A}(P) \mid=\varphi$ holds. More precisely (where $m$ is the maximal arity of a relation from the signature of $P)$ :

(1) FOMC(iSAb) can be decided in space

$$
g_{P}^{\prime}\left(2^{|\varphi|}\right)^{O(m)} \cdot \exp \left(2,|P|^{O(1)}\right) \leq \exp \left(2,|P|^{O(1)}+|\varphi|\right) .
$$

(2) $\mathrm{FOMC}(\mathrm{SAb})$ can be decided in space

$$
\exp (3, O(|P|)+\log (|\varphi|)) .
$$

(3) $\mathrm{FOMC}(\mathrm{iTAb})$ can be decided in time

$$
\exp \left(1, g_{P}^{\prime}\left(2^{|\varphi|}\right)^{O(m)} \cdot \exp \left(3,|P|^{O(1)}\right)\right) \leq \exp \left(4,|P|^{O(1)}+\log (|\varphi|)\right) .
$$

Proof. The decidability follows immediately from Theorem 2.4 and Prop. 2.10(a).

We first give the proof for injective string automatic presentations. By Prop. 3.3 it suffices to compute the Boolean value $\varphi_{\emptyset}$. Recall the inductive definition of $\varphi_{\sigma}$ from Def. 3.2 that we now translated into an algorithm for computing $\varphi_{\emptyset}$.

First note that such an algorithm has to handle potential $(d, k)$-spheres for $1 \leq k \leq d \leq|\varphi|$ ( $d$ is the quantifier rank of $\varphi$ ) that are realizable in $\mathcal{A}(P)$. The number of nodes of a potential $(d, k)$-sphere realizable in $\mathcal{A}(P)$ is bounded by $k \cdot g_{P}^{\prime}\left(2^{d}\right) \in g_{P}^{\prime}\left(2^{d}\right)^{O(1)}$ since $k \leq d<2^{d} \leq$ $g_{P}^{\prime}\left(2^{d}\right)$. The number of relations of $\mathcal{A}(P)$ is bounded by $|P|$. Hence, any potential $(d, k)$-sphere can be described by $|P| \cdot g_{P}^{\prime}\left(2^{d}\right) O(m)$ many bits.

Note that the set of $(d, k)$-spheres with $0 \leq k \leq d$ (ordered by the extension relation $\preceq$ ) forms a tree of depth $d+1$. The algorithm visits the nodes of this tree in a depth-first manner (and descents when unraveling an existential quantifier). Hence we have to store $d+1$ many spheres. For this, the algorithm needs space $(d+1) \cdot|P| \cdot g_{P}^{\prime}\left(2^{d}\right)^{O(m)}=|P| \cdot g_{P}^{\prime}\left(2^{d}\right)^{O(m)}$.

Moreover, during the unraveling of a quantifier, the algorithm has to check realizability of a potential $(d, k)$-sphere for $1 \leq k \leq d \leq|\varphi|$. Any such sphere has at most $g_{P}^{\prime}\left(2^{d}\right)^{O(1)}$ many elements and the degree $\delta$ of $\mathcal{A}$ is bounded by $\exp \left(1,|P|^{O(1)}\right)$ by Prop. 2.11. Hence, by Lemma 3.5, realizability can be checked in space $g_{P}^{\prime}\left(2^{d}\right)^{O(m)} \cdot|P|^{2} \cdot \exp \left(2,|P|^{O(1)}\right) \leq$ $g_{P}^{\prime}\left(2^{|\varphi|}\right)^{O(m)} \cdot \exp \left(2,|P|^{O(1)}\right)$.

At the end, we have to check whether a tuple $\bar{b}$ satisfies an atomic formula $\psi(\bar{y})$, which is trivial. In total, the algorithm runs in space

$$
|P| \cdot g_{P}^{\prime}\left(2^{|\varphi|}\right)^{O(m)}+g_{P}^{\prime}\left(2^{|\varphi|}\right)^{O(m)} \cdot \exp \left(2,|P|^{O(1)}\right) \leq g_{P}^{\prime}\left(2^{|\varphi|}\right)^{O(m)} \cdot \exp \left(2,|P|^{O(1)}\right) .
$$


Recall that $g_{\mathcal{A}}^{\prime}\left(2^{|\varphi|}\right) \leq \delta^{2^{|\varphi|}}$ and $\delta \leq 2^{|P|^{O(1)}}$ by Prop. 2.11. Since also $m \leq|P|$, we obtain

$$
\begin{aligned}
g_{P}^{\prime}\left(2^{|\varphi|}\right)^{O(m)} \cdot \exp \left(2,|P|^{O(1)}\right) & \leq \exp \left(1,|P|^{O(1)} \cdot 2^{|\varphi|} \cdot O(m)\right) \cdot \exp \left(2,|P|^{O(1)}\right) \\
& \leq \exp \left(2,|P|^{O(1)}+|\varphi|\right) .
\end{aligned}
$$

This completes the consideration for injective string automatic presentations.

If $P$ is just automatic, we can transform it into an equivalent injective automatic presentation which increases the size exponentially by Lemma 2.6. Hence, replacing $|P|$ by $2^{O(|P|)}$ yields the space bound.

Next, we consider injective tree automatic presentations. The algorithm is the same, i.e., it parses the tree of all potential $(d, k)$-spheres and checks them for realizability. Note that the number of potential $(d, k)$-spheres is in $\exp \left(1,|P| \cdot g_{P}^{\prime}\left(2^{d}\right)^{O(m)}\right)$. By Prop. 2.11, the degree $\delta$ is bounded by $\exp \left(2,|P|^{O(1)}\right)$. Hence, by Lemma 3.5, the realizability of any potential $(d, k)$ sphere can be checked in time

$$
\exp \left(1, g_{P}^{\prime}\left(2^{d}\right)^{O(m)} \cdot|P|^{2} \cdot \exp \left(3,|P|^{O(1)}\right)\right) \leq \exp \left(1, g_{P}^{\prime}\left(2^{|\varphi|}\right)^{O(m)} \cdot \exp \left(3,|P|^{O(1)}\right)\right) .
$$

Recall that $g_{P}^{\prime}\left(2^{|\varphi|}\right) \leq \delta^{2^{|\varphi|}}$ and $\delta \leq \exp \left(2,|P|^{O(1)}\right)$ by Prop. 2.11, Since also $m \leq|P|$, we obtain

$$
\begin{aligned}
g_{P}^{\prime}\left(2^{|\varphi|}\right)^{O(m)} \cdot \exp \left(3,|P|^{O(1)}\right) & \leq \exp \left(2,|P|^{O(1)}\right)^{2^{|\varphi|} \cdot O(|P|)} \cdot \exp \left(3,|P|^{O(1)}\right) \\
& =\exp \left(2,|P|^{O(1)}+|\varphi|\right) \cdot \exp \left(3,|P|^{O(1)}\right) \\
& =\exp \left(3,|P|^{O(1)}+\log (|\varphi|)\right)
\end{aligned}
$$

Remark 3.7. Note that the above theorem does not give the complexity for FOMC(TAb), i.e., for arbitrary tree automatic presentations of bounded degree: Here, one can proceed as for string automatic presentations, i.e., make the presentation injective and refer to the above result on FOMC(iTAb) - this gives the decidability that we already know from Theorem 2.4 and Prop. 2.10. At present, we cannot compare the complexity of this new algorithm with the nonelementary one from Theorem 2.4 since the size of the injective presentation is not known.

We derive a number of consequences on the uniform and non-uniform complexity of the first-order theories of string/tree automatic structures of bounded degree. The first one concerns the uniform model checking problems and is a direct consequence of the above theorem.

Corollary 3.8. The following holds:

- The model checking problem FOMC(iSAb) belongs to 2EXPSPACE.

- The model checking problem FOMC(SAb) belongs to 3EXPSPACE.

- The model checking problem FOMC(iTAb) belongs to 4EXPTIME.

Next we concentrate on the non-uniform complexity, where the structure is fixed. For string automatic structures, we do not get a better upper bound in this case (statement (i) below) except in case of polynomial growth (statement (ii) below). 
Corollary 3.9. Let $\mathcal{A}$ be a string automatic structure of bounded degree.

(i) Then $\operatorname{FOTh}(\mathcal{A})$ belongs to 2EXPSPACE.

(ii) If $\mathcal{A}$ has polynomial growth then $\operatorname{FOTh}(\mathcal{A})$ belongs to EXPSPACE.

Proof. Since $\mathcal{A}$ is string automatic, it has a fixed injective string automatic presentation $P$, i.e., $|P|$ and $m$ are fixed constants. Hence the result follows immediately from (1) in Theorem 3.6.

Now suppose that $\mathcal{A}$ has polynomial growth, i.e., $g_{\mathcal{A}}^{\prime}(x) \in x^{O(1)}$. Then, again, the claim follows immediately from (1) in Theorem [3.6, since $g_{\mathcal{A}}^{\prime}\left(2^{|\varphi|}\right)^{O(m)} \leq 2^{O(|\varphi|)}$.

The last consequence of Theorem 3.6 concerns tree automatic structures. Here, we can improve the upper bound from Theorem 3.6 for the non-uniform case by one exponent. In case of polynomial growth, we can save yet another exponent:

Corollary 3.10. Let $\mathcal{A}$ be a tree automatic structure of bounded degree.

(i) Then $\operatorname{FOTh}(\mathcal{A})$ belongs to 3EXPTIME.

(ii) If $\mathcal{A}$ has polynomial growth then $\operatorname{FOTh}(\mathcal{A})$ belongs to 2EXPTIME.

Proof. Since $\mathcal{A}$ is tree automatic, it has a fixed injective tree automatic presentation $P$. Hence, again, the first claim follows immediately from (3) in Theorem 3.6 .

Now suppose that $\mathcal{A}$ has polynomial growth, i.e., $g_{\mathcal{A}}^{\prime}(x) \in x^{O(1)}$. Then the claim follows since

$$
\exp \left(1, g_{\mathcal{A}}^{\prime}\left(2^{|\varphi|}\right)^{O(m)}\right) \leq \exp \left(1,2^{O(|\varphi|)}\right)=\exp (2, O(|\varphi|)),
$$

implying that the problem belongs to 2EXPTIME.

Two observations on the growth function We complement this section with a short excursion into the field of growth functions of automatic structures. The two results to be reported indicate that these growth functions do not behave as nicely as one would wish. Fortunately, these negative findings are of no importance to our main concerns.

Recall that the growth rate of a regular language is either bounded by a polynomial from above or by an exponential function from below and that it is decidable which of these cases applies. The next lemmas show that the analogous statements for growth functions of string automatic structures are false.

Lemma 3.11. There is a string automatic graph of intermediate growth (i.e., the growth is neither exponential nor polynomial).

Proof. Let $L=\{0,1\}^{*} \$\{0,1\}^{*}$ and let $E$ be

$$
\left\{(u \$ b v, u b \$ v) \mid u, v \in\{0,1\}^{*}, b \in\{0,1\}\right\} \cup\left\{(u \$, \$ u b) \mid u \in\{0,1\}^{*}, b \in\{0,1\}\right\} .
$$

Then $T=(L, E)$ is a string automatic tree obtained from the complete binary tree $\$\{0,1\}^{*}$ by adding a path of length $n$ between $u$ and $u b$ for $u \in\{0,1\}^{n}$ and $b \in\{0,1\}$. Hence, a path of length $n$ starting in the root $\$$ of $T$ branches at distance $0,2,5,10, \ldots, i^{2}+1, \ldots,\lfloor\sqrt{n-1}\rfloor^{2}+1$ from the root. Hence, for the growth function $g_{T}$ we obtain the following estimate:

$$
g_{T}(n) \in \sum_{i=0}^{\Theta(\sqrt{n})}(i+1) \cdot 2^{i}=\Theta(\sqrt{n}) \cdot 2^{\Theta(\sqrt{n})}=2^{\Theta(\sqrt{n})}
$$


Lemma 3.12. It is undecidable whether a string automatic graph of bounded degree has polynomial growth.

Proof. We show the undecidability by a reduction of the halting problem (with empty input) for Turing machines. So let $N$ be a Turing machine. We can transform $N$ into a deterministic reversible Turing machine $M$ such that:

(i) $N$ halts on empty input if and only if $M$ does so.

(ii) $M$ does not allow infinite sequences of backwards steps (i.e., there are no configurations $c_{i}$ with $c_{i+1} \vdash_{M} c_{i}$ for all $i \in \mathbb{N}$ ), see also [21] for a similar construction.

Let $C$ be the set of configurations of $M$ (a regular set) and $c_{0}$ the initial configuration with empty input. Now define $L=(\{0,1\} C)^{+}$(we assume that 0 and 1 do not belong to the alphabet of $C$ ) and

$$
\begin{aligned}
E= & \left\{\left(u a c, u a c^{\prime}\right) \mid u \in L \cup\{\varepsilon\}, a \in\{0,1\}, c, c^{\prime} \in C, c \vdash_{M} c^{\prime}\right\} \cup \\
& \left\{\left(u a c, u a c b c_{0}\right) \mid u \in L \cup\{\varepsilon\}, a, b \in\{0,1\}, c \in C \text { is halting }\right\} .
\end{aligned}
$$

Then $(L, E)$ is an automatic directed graph. Since $M$ is reversible, it is a forest of rooted trees (by (ii)).

First suppose there are configurations $c_{1}, c_{2}, \ldots, c_{n}$ with $c_{i-1} \vdash_{M} c_{i}$ for $1 \leq i \leq n$ such that $c_{n}$ is halting. Then the set $0\left(c_{n}\{0,1\}\right)^{*}\left\{c_{0}, c_{1}, \ldots, c_{n}\right\}$ forms an infinite tree in $(L, E)$. Any branch in this tree branches every $n$ steps. Hence $(L, E)$ has exponential growth.

Now assume that $c_{0}$ is the starting point of an infinite computation. Let $T$ be any tree in the forest $(L, E)$. Then its root is of the form $u a c \in L$ with $u \in L \cup\{\varepsilon\}, a \in\{0,1\}$, and $c \in C$ such that $c$ is no successor configuration of any other configuration. There are two possibilities:

1. The configuration $c$ is the starting configuration of an infinite computation of $M$. Then $T$ is an infinite path.

2. There is a halting configuration $c^{\prime}$ and $n \in \mathbb{N}$ with $c \vdash_{M}^{n} c^{\prime}$. Then $T$ starts with a path of length $n$. The final node of this path has two children, namely $u a c^{\prime} 0 c_{0}$ and $u a c^{\prime} 1 c_{0}$. But, since $M$ does not halt on the empty input, each of these nodes is the root of an infinite path.

Thus, in this case $(L, E)$ has polynomial (even linear) growth.

\section{Lower bounds}

In this section, we will prove that the upper complexity bounds for the non-uniform problems (Cor. 3.9 and Cor. 3.10) are sharp. This will imply that the complexity of the uniform problem for injective string automatic presentations from Theorem [3.6 is sharp as well.

For a binary relation $r$ and $m \in \mathbb{N}$ we denote with $r^{m}$ the $m$-fold composition of $r$. Then the following lemma is folklore.

Lemma 4.1. Let the signature $\mathcal{S}$ contain a binary symbol $r$. From a given number $m$ (encoded unary), we can construct in linear time a formula $\varphi_{m}(x, y)$ such that for every $\mathcal{S}$-structure $\mathcal{A}$ and all elements $a, b \in \mathcal{A}$ we have: $(a, b) \in r^{2^{m}}$ if and only if $\mathcal{A}=\varphi_{m}(a, b)$. 
Proof. Let $\varphi_{0}(x, y)=r(x, y)$ and, for $m>0$ define

$$
\varphi_{m}(x, y)=\exists z \forall x^{\prime}, y^{\prime}\left(\left(\left(x^{\prime}=x \wedge y^{\prime}=z\right) \vee\left(x^{\prime}=z \wedge y^{\prime}=y\right)\right) \rightarrow \varphi_{m-1}\left(x^{\prime}, y^{\prime}\right)\right) .
$$

For a bit string $u=a_{1} \cdots a_{m}\left(a_{i} \in\{0,1\}\right)$ let val $(u)=\sum_{i=0}^{m-1} a_{i+1} 2^{i}$ be the integer value represented by $u$. Vice versa, for $0 \leq i \leq 2^{m}-1$ let $\operatorname{bin}_{m}(i) \in\{0,1\}^{m}$ be the unique string with $\operatorname{val}\left(\operatorname{bin}_{m}(i)\right)=i$.

Theorem 4.2. There exists a fixed string automatic structure $\mathcal{A}$ of bounded degree such that $\operatorname{FOTh}(\mathcal{A})$ is 2EXPSPACE-hard.

Proof. Let $M$ be a fixed Turing machine with a space bound of $\exp (2, n)$ such that $M$ accepts a 2EXPSPACE-complete language; such a machine exists by standard arguments. Let $\Gamma$ be the tape alphabet, $\Sigma \subseteq \Gamma$ be the input alphabet, and $Q$ be the set of states. The initial (resp. accepting) state is $q_{0} \in Q$ (resp. $q_{f} \in Q$ ), the blank symbol is $\square \in \Gamma \backslash \Sigma$. Let $\Omega=Q \cup \Gamma$. A configuration of $M$ is described by a string from $\Gamma^{*} Q \Gamma^{+} \subseteq \Omega^{+}$(later, symbols of configurations will be preceded with additional counters). For two configurations $u$ and $v$ with $|u|=|v|$ we write $u \vdash_{M} v$ if $u$ can evolve with a single $M$-transition into $v$. Note that there exists a relation $\alpha_{M} \subseteq \Omega^{3} \times \Omega^{3}$ such that for all configurations $u=a_{1} \cdots a_{m}$ and $v=b_{1} \cdots b_{m}\left(a_{i}, b_{i} \in \Omega\right)$ we have

$$
u \vdash_{M} v \Longleftrightarrow \forall i \in\{1, \ldots, m-2\}:\left(a_{i} a_{i+1} a_{i+2}, b_{i} b_{i+1} b_{i+2}\right) \in \alpha_{M} .
$$

Let $\Delta=\{0,1, \#\} \cup \Omega$, and let $\pi: \Delta \rightarrow \Omega \cup\{\#\}$ be the projection morphism with $\pi(a)=a$ for $a \in \Omega \cup\{\#\}$ and $\pi(0)=\pi(1)=\varepsilon$. For $m \in \mathbb{N}$, a string $x \in \Delta^{*}$ is an accepting $2^{m}$-computation if $x$ can be factorized as $x=x_{1} \# x_{2} \# \cdots x_{n} \#$ for some $n \geq 1$ such that the following holds:

- For every $1 \leq i \leq n$ there exist $a_{i, 0}, \ldots, a_{i, 2^{m}-1} \in \Omega$ such that $x_{i}=\prod_{j=0}^{2^{m}-1} \operatorname{bin}_{m}(j) a_{i, j}$.

- For every $1 \leq i \leq n, \pi\left(x_{i}\right) \in \Gamma^{*} Q \Gamma^{+}$.

$-\pi\left(x_{1}\right) \in q_{0} \Sigma^{*} \square^{*}$ and $\pi\left(x_{n}\right) \in \Gamma^{*} q_{f} \Gamma^{+}$

- For every $1 \leq i<n, \pi\left(x_{i}\right) \vdash_{M} \pi\left(x_{i+1}\right)$.

From $M$ we now construct a fixed string automatic structure $\mathcal{A}$ of bounded degree. We start with the following regular language $U_{0}$ :

$$
\begin{aligned}
U_{0}= & \pi^{-1}\left(\left(\Gamma^{*} Q \Gamma^{+} \#\right)^{*}\right) \cap \\
& \left(0^{+} \Omega\left(\{0,1\}^{+} \Omega\right)^{*} 1^{+} \Omega \#\right)^{+} \cap \\
& 0^{+} q_{0}\left(\{0,1\}^{+} \Sigma\right)^{*}\left(\{0,1\}^{+} \square\right)^{*} \# \Delta^{*} \cap \\
& \Delta^{*} q_{f}(\Delta \backslash\{\#\})^{*} \#
\end{aligned}
$$

A string $x \in U_{0}$ is a candidate for an accepting $2^{m}$-computation of $M$. With (11) we describe the basic structure of such a computation, it consists of a list of configurations separated by \#. Moreover, every symbol in a configuration is preceded by a bit string, which represents a counter. By (12) every counter is non-empty, the first symbol in a configuration is preceded by a counter from $0^{+}$, the last symbol is preceded by a counter from $1^{+}$. Moreover, by (13), the first configuration is an initial configuration, whereas by (14), the last configuration is accepting (i.e. the current state is $q_{f}$ ).

For the further considerations, let us fix some $x \in U_{0}$. Hence, we can factorize $x$ as $x=x_{1} \# x_{2} \# \cdots x_{n} \#$ such that: 
- For every $1 \leq i \leq n$, there exist $m_{i} \geq 1, a_{i, 0}, \ldots, a_{i, m_{i}} \in \Omega$ and counters $u_{i, 0}, \ldots, u_{i, m_{i}} \in$ $\{0,1\}^{+}$such that $x_{i}=\prod_{j=0}^{m_{i}} u_{i, j} a_{i, j}$.

- For every $1 \leq i \leq n, u_{i, 0} \in 0^{+}, u_{i, m_{i}} \in 1^{+}$, and $\pi\left(x_{i}\right) \in \Gamma^{*} Q \Gamma^{+}$.

$-\pi\left(x_{1}\right) \in q_{0} \Sigma^{*} \square^{*}$ and $\pi\left(x_{n}\right) \in \Gamma^{*} q_{f} \Gamma^{+}$

We next want to construct, from $m \in \mathbb{N}$, a small formula expressing that $x$ is an accepting $2^{m}$-computation. To achieve this, we add some structure around strings from $U_{0}$. Then the formula we are seeking has to ensure two facts:

(a) The counters behave correctly, i.e. for all $1 \leq i \leq n$ and $0 \leq j \leq m_{i}$, we have $\left|u_{i, j}\right|=m$ and if $j<m_{i}$, then $\operatorname{val}\left(u_{i, j+1}\right)=\operatorname{val}\left(u_{i, j}\right)+1$. Note that this enforces $m_{i}=2^{m}-1$ for all $1 \leq i \leq n$.

(b) For two successive configurations, the second one is the successor configuration of the first one with respect to the machine $M$, i.e., $\pi\left(x_{i}\right) \vdash_{M} \pi\left(x_{i+1}\right)$ for all $1 \leq i<n$.

In order to achieve (a), we introduce the following three binary relations; it is straightforward to exhibit 2-dimensional automata for these relations:

$$
\begin{aligned}
& \delta=\left\{(w, w \otimes w) \mid w \in U_{0}\right\} \\
& \sigma_{0}=\left\{\left(\left(0 v_{1} \# 0 v_{2} \# \cdots 0 v_{n} \#\right) \otimes w,\left(v_{1} 0 \# v_{2} 0 \# \cdots v_{n} 0 \#\right) \otimes w\right) \mid\right. \\
&\left.w \in U_{0}, v_{1}, \ldots, v_{n} \in(\Delta \backslash\{\#\})^{*}\right\} \\
& \sigma_{\Omega}=\left\{\left(\left(a_{1} v_{1} \# a_{2} v_{2} \# \cdots a_{n} v_{n} \#\right) \otimes w,\left(v_{1} a_{1} \# v_{2} a_{2} \# \cdots v_{n} a_{n} \#\right) \otimes w\right) \mid\right. \\
&\left.w \in U_{0}, a_{1}, \ldots, a_{n} \in \Omega, v_{1}, \ldots, v_{n} \in(\Delta \backslash\{\#\})^{*}\right\}
\end{aligned}
$$

Hence, $\delta$ just duplicates a string from $U_{0}$ and $\sigma_{0}$ cyclically rotates every configuration to the left for one symbol, provided the first symbol is 0 , whereas $\sigma_{\Omega}$ rotates symbols from $\Omega$. Moreover, let $U_{1}$ be the following regular language over $\Delta^{*} \otimes \Delta^{*}$ :

$$
U_{1}=\left(\left(\left\{u \otimes v\left|u, v \in\{0,1\}^{+},\right| u|=| v \mid, \operatorname{val}(u)=\operatorname{val}(v)+1 \bmod 2^{|u|}\right\}(\Omega \times \Omega)\right)^{+}(\#, \#)\right)^{+}
$$

Clearly, $U_{1}$ is a regular language. The crucial fact is the following:

Fact 1. For every $m \in \mathbb{N}$, the following two properties are equivalent (recall that $x \in U_{0}$ ):

- There exist $y_{1}, y_{2}, y_{3} \in \Delta^{*} \otimes \Delta^{*}$ such that $\delta\left(x, y_{1}\right), \sigma_{0}^{m}\left(y_{1}, y_{2}\right), \sigma_{\Omega}\left(y_{2}, y_{3}\right), y_{3} \in U_{1}$.

- For all $1 \leq i \leq n$ and $0 \leq j \leq m_{i}$, we have $\left|u_{i, j}\right|=m$ and if $j<m_{i}$, then $\operatorname{val}\left(u_{i, j+1}\right)=$ $\operatorname{val}\left(u_{i, j}\right)+1$.

Assume now that $x \in U_{0}$ satisfies one (and hence both) of the two properties from Fact 1 for some $m$. It follows that $m_{i}=2^{m}-1$ for all $1 \leq i \leq n$ and

$$
x=x_{1} \# x_{2} \# \cdots x_{n} \#, \text { where } x_{i}=\prod_{j=0}^{2^{m}-1} \operatorname{bin}_{m}(j) a_{i, j} \text { for every } 1 \leq i \leq n .
$$

In order to establish (b) we need additional structure. The idea is, for every counter value $0 \leq$ $j<2^{m}$, to have a word $y_{j}$ that coincides with $x$, but has all the occurrences of $\operatorname{bin}_{m}(j)$ marked. Then an automaton can check that successive occurrences of the counter $\operatorname{bin}_{m}(j)$ obey the transition condition of the Turing machine. There are two problems with this approach: first, in order to relate $x$ and $y_{j}$, we would need a binary relation of degree $2^{m}$ (for arbitrary $m$ ) 
and, secondly, an automaton cannot mark all the occurrences of $\operatorname{bin}_{m}(j)$ at once (for some $j)$. In order to solve these problems, we introduce a binary relation $\mu$, which for every $x \in U_{0}$ as in (15) generates a binary tree of depth $m$ with root $x$; this will be the only relation in our string automatic structure that causes exponential growth. This relation will mark in $x$ every occurrence of an arbitrary counter. For this, we need two copies $\overline{0}$ and $\underline{0}$ of 0 as well as two copies $\overline{1}$ and $\underline{1}$ of 1 . For $b \in\{0,1\}$, define the mapping

$$
f_{b}:\{\underline{0}, \overline{0}, \underline{1}, \overline{1}\}^{*}\{0,1\}^{+} \rightarrow\{\underline{0}, \overline{0}, \underline{1}, \overline{1}\}^{+}\{0,1\}^{*}
$$

as follows (where $u \in\{\underline{0}, \overline{0}, \underline{1}, \overline{1}\}^{*}, c \in\{0,1\}$, and $v \in\{0,1\}^{*}$ ):

$$
f_{b}(u c v)= \begin{cases}u \underline{c} v & \text { if } b \neq c \\ u \bar{c} v & \text { if } b=c\end{cases}
$$

We extend $f_{b}$ to a function on $\left(\left(\{\underline{0}, \overline{0}, \underline{1}, \overline{1}\}^{*}\{0,1\}^{+} \Omega\right)^{+} \#\right)^{*}$ as follows: Let $w=w_{1} a_{1} \cdots w_{\ell} a_{\ell}$ with $w_{i} \in\{\underline{0}, \overline{0}, \underline{1}, \overline{1}\}^{*}\{0,1\}^{+}$and $a_{i} \in \Omega \cup \Omega \#$. Then $f_{b}(w)=f_{b}\left(w_{1}\right) a_{1} \cdots f_{b}\left(w_{\ell}\right) a_{\ell}$; this mapping can be computed with a synchronized transducer. Hence, the relation

$$
\mu=f_{0} \cup f_{1}=\left\{\left(u, f_{b}(u)\right) \mid u \in\left(\left(\{\underline{0}, \overline{0}, \underline{1}, \overline{1}\}^{*}\{0,1\}^{+} \Omega\right)^{+} \#\right)^{*}, b \in\{0,1\}\right\}
$$

can be recognized by a 2-dimensional automaton.

Let $x \in U_{0}$ as in (15), let the word $y$ be obtained from $x$ by overlining or underlining each bit in $x$, and let $u \in\{0,1\}^{m}$ be some counter. We say the counter $u$ is marked in $y$ if every occurrence of the counter $u$ is marked by overlining each bit, whereas all other counters contain at least one underlined bit.

Fact 2. Let $x \in U_{0}$ be as in (15).

- For all counters $u \in\{0,1\}^{m}$, there exists a unique word $y$ with $(x, y) \in \mu^{m}$ such that the counter $u$ is marked in $y$.

- If $(x, y) \in \mu^{m}$, then there exists a unique counter $u \in\{0,1\}^{m}$ such that $u$ is marked in $y$.

Now, we can achieve our final goal, namely checking whether two successive configurations in $x \in U_{0}$ represent a transition of the machine $M$. Let the counter $u \in\{0,1\}^{m}$ be marked in $y$. We describe a finite automaton $A_{2}$ that checks on the string $y$, whether at position $\operatorname{val}(u)$ successive configurations in $x$ are "locally consistent". The automaton $A_{2}$ searches for the first marked counter in $y$. Then it stores the next three symbols $a_{1}, a_{2}, a_{3}$ from $\Omega$ (if the separator \# is seen before, then only one or two symbols may be stored), walks right until it finds the next marked counter, reads the next three symbols $b_{1}, b_{2}, b_{3}$ from $\Omega$, and checks whether $\left(a_{1} a_{2} a_{3}, b_{1} b_{2} b_{3}\right) \in \alpha_{M}$, where $\alpha_{M}$ is from (10). If this is not the case, the automaton will reject, otherwise it will store $b_{1} b_{2} b_{3}$ and repeat the procedure described above. Let $U_{2}=L\left(A_{2}\right)$. Together with Fact 1 and 2, the behavior of $A_{2}$ implies that for all $x \in U_{0}$ and all $m \in \mathbb{N}, x$ represents an accepting $2^{m}$-computation of $M$ if and only if

$$
\exists y_{1}, y_{2}, y_{3}\left(\delta\left(x, y_{1}\right) \wedge \sigma_{0}^{m}\left(y_{1}, y_{2}\right) \wedge \sigma_{\Omega}\left(y_{2}, y_{3}\right) \wedge y_{3} \in U_{1}\right) \wedge \forall y\left(\mu^{m}(x, y) \rightarrow y \in U_{2}\right) .
$$

Let us now fix some input $w=a_{1} a_{2} \cdots a_{n} \in \Sigma^{*}$ with $|w|=n$, and let $a_{n+1}=\square$ and $m=2^{n}$. Thus, $w$ is accepted by $M$ if and only if there exists an accepting $2^{m}$-computation $x$ such that in the first configuration of $x$, the tape content is of the form $w \square^{+}$. It remains to add some 
structure that allows us to express the latter by a formula. But this is straightforward: Let $\triangleright$ be a new symbol and let $\Pi=\Delta \cup\{\underline{0}, \overline{0}, \underline{1}, \overline{1}, \triangleright\}$; this is our final alphabet. Define the binary relations $\iota_{0,1}$ and $\iota_{a}(a \in \Omega)$ as follows:

$$
\begin{aligned}
\iota_{0,1} & =\left\{(u \triangleright a v, u a \triangleright v) \mid a \in\{0,1\}, u, v \in \Delta^{*}, u a v \in U_{0}\right\} \cup\left\{(0 v, 0 \triangleright v) \mid v \in \Delta^{*}, 0 v \in U_{0}\right\} \\
\iota_{a} & =\left\{(u \triangleright a v, u a \triangleright v) \mid u, v \in \Delta^{*}, u a v \in U_{0}\right\} .
\end{aligned}
$$

Then, $\mathcal{A}=\left(\Pi^{*} \cup\left(\Pi^{*} \otimes \Pi^{*}\right), \delta, \sigma_{0}, \sigma_{\Omega}, \mu, \iota_{0,1},\left(\iota_{a}\right)_{a \in \Omega}, U_{0}, U_{1}, U_{2}\right)$ is a string automatic structure of bounded degree such that $w$ is accepted by $M$ if and only if the following formula is true in $\mathcal{A}$ :

$$
\exists x \in U_{0}\left\{\begin{array}{l}
\exists y_{1}, y_{2}, y_{3}\left(\delta\left(x, y_{1}\right) \wedge \sigma_{0}^{m}\left(y_{1}, y_{2}\right) \wedge \sigma_{\Omega}\left(y_{2}, y_{3}\right) \wedge y_{3} \in U_{1}\right) \wedge \\
\forall y\left(\mu^{m}(x, y) \rightarrow y \in U_{2}\right) \wedge \\
\exists y_{0}, z_{0}, \ldots, y_{n+1}, z_{n+1}\left(\iota_{0,1}^{m}\left(x, y_{0}\right) \wedge \iota_{q_{0}}\left(y_{0}, z_{0}\right) \wedge \bigwedge_{i=1}^{n+1} \iota_{0,1}^{m}\left(z_{i-1}, y_{i}\right) \wedge \iota_{a_{i}}\left(y_{i}, z_{i}\right)\right)
\end{array}\right\}
$$

By Lemma 4.1 we can compute in time $O(\log (m))=O(n)$ an equivalent formula over the signature of $\mathcal{A}$. This concludes the proof.

The following theorem, which proves an analogous result for tree automatic structures, uses alternating Turing machines, see [6 26] for more details. Roughly speaking, an alternating Turing machine is a nondeterministic Turing machine, where the set of states is partitioned into accepting, existential, and universal states. A configuration is accepting, if either (i) the current state is accepting, or (ii) the current state is existential and at least one successor configuration is accepting, or (iii) the current state is universal and every successor configuration is accepting. By [6], $k$ EXPTIME is the set of all problems that can be accepted in space $\exp \left(k-1, n^{O(1)}\right)$ on an alternating Turing machine (for all $k \geq 1$ ).

Theorem 4.3. There exists a fixed tree automatic structure $\mathcal{A}$ of bounded degree such that $\operatorname{FOTh}(\mathcal{A})$ is 3EXPTIME-hard.

Proof. Let $M$ be a fixed alternating Turing machine with a space bound of $\exp (2, n)$ such that $M$ accepts a 3EXPTIME-complete language. W.l.o.g. every configuration, where the current state is either existential or universal has exactly two successor configurations. Let $\Sigma, \Gamma, Q$, and $\Omega$ have the same meaning as in the previous proof. Moreover, let $\Delta=\Omega \cup\left\{0,1, \#_{\exists}, \#_{\forall}\right\}$.

The idea is that a binary tree $x$ over the alphabet $\Delta$ can encode a computation tree for some input. Configurations can be encoded by linear chains over the alphabet $\Omega \cup\{0,1\}$ as in the previous proof. The separator symbol $\#_{\exists}$ is used to separate an existential configuration from a successor configuration, whereas the separator symbol $\#_{\forall}$ is used to separate a universal configuration from its two successor configurations. Hence, a $\#_{\exists}{ }_{-}$labeled node has exactly one child, whereas a $\#_{\forall}$-labeled node has exactly two children. Checking whether the counters behave correctly can be done similarly to the previous proof by introducing binary relations $\sigma_{0}$ and $\sigma_{\Omega}$, which rotate symbols within configurations. Remember that in our tree encoding, configurations are just long chains. Also the marking of some specific counter can be done in the same way as before. Finally, having marked some specific counter allows to check with a top-down tree automaton, whether the tree $x$ represents indeed a valid computation tree. Of 
course, the tree automaton has to check whether the current configuration is existential or universal. In case of a universal configuration, the automaton branches at the next separator symbol $\#_{\forall}$. If e.g. the current configuration is universal but the next separator symbol is $\#_{\exists}$, then the automaton rejects the tree.

The proof of the next result is in fact a simplification of the proof of Theorem 4.2, since we do not need counters.

Theorem 4.4. There exists a fixed string automatic structure $\mathcal{A}$ of bounded degree and polynomial growth (in fact linear growth) such that $\operatorname{FOTh}(\mathcal{A})$ is EXPSPACE-hard.

Proof. Let $M$ be a fixed Turing machine with a space bound of $2^{n}$ such that $M$ accepts an EXPSPACE-complete language. Let $\Sigma, \Gamma, Q, q_{0}, q_{f}, \square$, and $\Omega$ have the usual meaning. Let $\Delta=\{\#\} \cup \Omega$. This time, for $m \in \mathbb{N}$, an accepting $m$-computation is a string $x_{1} \# x_{2} \# \cdots x_{n} \#$, where $x_{1}, \ldots, x_{n} \in \Gamma^{*} Q \Gamma^{+}$are configurations with $\left|x_{i}\right|=m(1 \leq i \leq n), x_{i} \vdash_{M} x_{i+1}$ $(1 \leq i<n), x_{1} \in q_{0} \Sigma^{*} \square^{*}$, and $x_{n} \in \Gamma^{*} q_{f} \Gamma^{+}$. Let $U_{0}$ be the fixed regular language

$$
U_{0}=\left(\Gamma^{*} Q \Gamma^{+} \#\right)^{+} \cap q_{0} \Sigma^{*} \square^{*} \# \Delta^{*} \cap \Delta^{*} q_{f}(\Delta \backslash\{\#\})^{*} \# .
$$

The following binary relations $\delta$ and $\sigma_{\Omega}$ can be easily recognized by 2-dimensional automata:

$$
\begin{aligned}
\delta & =\left\{(w, w \otimes w) \mid w \in U_{0}\right\} \\
\sigma_{\Omega} & =\left\{(a v \otimes w, v a \otimes w) \mid w \in U_{0}, a \in \Omega, v \in \Delta^{*}\right\}
\end{aligned}
$$

Moreover, let $U_{1}$ be the following regular language over $\Delta^{*} \otimes \Delta^{*}$ :

$$
U_{1}=\left\{\# u \otimes v \#\left|u, v \in \Omega^{+},\right| u|=| v \mid, v \vdash_{M} u\right\}^{+}\left\{\# u \otimes v \#\left|u, v \in \Omega^{+},\right| u|=| v \mid\right\} .
$$

Then, for every $x \in U_{0}$ and $m \in \mathbb{N}$ we have: $x$ is an accepting $m$-computation if and only if there exist $y_{1}, y_{2} \in \Delta^{*} \otimes \Delta^{*}$ such that $\delta\left(x, y_{1}\right), \sigma_{\Omega}^{m}\left(y_{1}, y_{2}\right)$, and $y_{2} \in U_{1}$.

Let us now fix some input $w=a_{1} \cdots a_{n} \in \Sigma^{*}$ with $|w|=n$, let $a_{n+1}=\square$, and let $m=2^{n}$. Thus, $w$ is accepted by $M$ if and only if there exists an accepting $m$-computation $x$ such that in the first configuration of $x$, the tape content is of the form $w \square^{+}$. It remains to add some structure that allows us to express the latter by a formula. This can be done similarly to the proof of Theorem 4.2 . Let $\Pi=\Delta \cup\{\triangleright\}$, where $\triangleright$ is a new symbol and define the binary relations $\iota_{a}(a \in \Sigma \cup\{\square\})$ as follows:

$$
\iota_{a}=\left\{\left(q_{0} a v, q_{0} a \triangleright v\right) \mid v \in \Delta^{*}, q_{0} a v \in U_{0}\right\} \cup\left\{(u \triangleright a v, u a \triangleright v) \mid u, v \in \Delta^{*}, u a v \in U_{0}\right\}
$$

Then, $\mathcal{A}=\left(\Pi^{*} \cup\left(\Delta^{*} \otimes \Delta^{*}\right), \delta, \sigma_{\Omega},\left(\iota_{a}\right)_{a \in \Sigma \cup\{\square\}}, U_{0}, U_{1}\right)$ is a fixed string automatic structure of bounded degree and linear growth. For the latter note that the Gaifman graph of $\mathcal{A}$ is just a disjoint union of cycles and finite paths (in fact, every node has degree at most 2). Moreover, $w$ is accepted by $M$ if and only if the following statement is true in $\mathcal{A}$ :

$$
\exists x \in U_{0}\left\{\begin{array}{l}
\exists y_{1}, y_{2}\left(\delta\left(x, y_{1}\right) \wedge \sigma_{\Omega}^{m}\left(y_{1}, y_{2}\right) \wedge y_{2} \in U_{1}\right) \wedge \\
\exists y_{0}, \ldots, y_{n}\left(\iota_{a_{1}}\left(x, y_{0}\right) \wedge \bigwedge_{i=1}^{n} \iota_{a_{i}}\left(y_{i-1}, y_{i}\right)\right)
\end{array}\right\} .
$$

By Lemma 4.1 this concludes the proof. 
The next result can be easily shown by combining the techniques from the proof of Theorem 4.3 and 4.4. We leave the details for the reader.

Theorem 4.5. There exists a fixed tree automatic structure $\mathcal{A}$ of bounded degree and polynomial growth (in fact linear growth) such that $\operatorname{FOTh}(\mathcal{A})$ is 2EXPTIME-hard.

\section{Bounded quantifier alternation depth}

In this section we prove some facts about first-order fragments of fixed quantifier alternation depth. These results will follow easily from the constructions in the preceding section.

For $n \geq 0, \Sigma_{n}$-formulas and $\Pi_{n}$-formulas are inductively defined as follows:

- A quantifier-free first-order formula is a $\Sigma_{0}$-formula as well as a $\Pi_{0}$-formula.

- If $\varphi\left(x_{1}, \ldots, x_{n}, y_{1}, \ldots, y_{m}\right)$ is a $\Sigma_{n}$-formula, then $\forall x_{1} \ldots \forall x_{n}: \varphi\left(x_{1}, \ldots, x_{n}, y_{1}, \ldots, y_{m}\right)$ is a $\Pi_{n+1}$-formula.

- If $\varphi\left(x_{1}, \ldots, x_{n}, y_{1}, \ldots, y_{m}\right)$ is a $\Pi_{n}$-formula, then $\exists x_{1} \ldots \exists x_{n}: \varphi\left(x_{1}, \ldots, x_{n}, y_{1}, \ldots, y_{m}\right)$ is a $\Sigma_{n+1}$-formula.

The $\Sigma_{n}$-theory $\Sigma_{n}$-FOTh $(\mathcal{A})$ of a structure $\mathcal{A}$ is the set of all $\Sigma_{n}$-formulas in $\operatorname{FOTh}(\mathcal{A})$; the $\Pi_{n}$-theory is defined analogously. For a class $\mathrm{C}$ of tree automatic presentations, the $\Sigma_{n}$-model checking problem $\Sigma_{n}$-FOMC(C) of $\mathrm{C}$ denotes the set of all pairs $(P, \varphi)$ where $P \in \mathrm{C}$, and $\varphi \in \Sigma_{n}-\operatorname{FOTh}(\mathcal{A}(P))$.

The following result can be found in [5]:

Theorem 5.1 (cf. [5]). The $\Sigma_{1}$-model checking problem $\Sigma_{1}$-FOMC(SA) for all string automatic presentations is in PSPACE. Moreover, there is a fixed string automatic structure with a PSPACE-complete $\Sigma_{1}$-theory.

From our construction in the proof of Theorem 4.4, we can slightly sharpen the lower bound in this theorem:

Theorem 5.2. There exists a fixed string automatic structure of bounded degree and linear growth with a PSPACE-complete $\Sigma_{1}$-theory.

Proof. Take the structure $\mathcal{A}$ from the proof of Theorem 4.4 and let $M$ be a fixed linear bounded automaton with a PSPACE-complete acceptance problem. If we replace the number $m$ in the formula (16) by the input length $n$, then (16) is equivalent to the following formula, which is equivalent to a $\Sigma_{1}$-formula:

$$
\exists x \in U_{0}\left\{\begin{array}{l}
\exists y_{0}, \ldots, y_{n+1}\left(\delta\left(x, y_{0}\right) \wedge \bigwedge_{i=0}^{n} \sigma_{\Omega}\left(y_{i}, y_{i+1}\right) \wedge y_{n+1} \in U_{1}\right) \wedge \\
\exists y_{1}, \ldots, y_{n}\left(\iota_{a_{1}}\left(x, y_{1}\right) \wedge \bigwedge_{i=2}^{n} \iota_{a_{i}}\left(y_{i-1}, y_{i}\right)\right)
\end{array}\right\} .
$$

This formula is true in $\mathcal{A}$ if and only if the linear bounded automaton accepts the input $w=a_{1} \cdots a_{n}$.

Let us now move on to $\Sigma_{2}$-formulas and structures of arbitrary growth: 
Theorem 5.3. The $\Sigma_{2}$-model checking problem $\Sigma_{2}$-FOMC(SA) for all string automatic presentations is in EXPSPACE. Moreover, there is a string automatic structure of bounded degree with an EXPSPACE-complete $\Sigma_{2}$-theory.

Proof. For the upper bound, let $P$ be a string automatic presentations of the automatic structure $\mathcal{A}(P)=\mathcal{A}$ and let

$$
\psi=\exists x_{1} \cdots \exists x_{n} \forall y_{1} \cdots \forall y_{m}: \varphi
$$

be a $\Sigma_{2}$-sentence. The sentence $\psi$ is equivalent to

$$
\exists x_{1} \cdots \exists x_{n} \neg \exists y_{1} \cdots \exists y_{m}: \neg \varphi .
$$

Negations in $\neg \varphi$ can be moved down to the level of atomic predicates. Then, we can built an $(n+m)$-dimensional automaton for $\neg \varphi$ with $\exp \left(1,|\psi|^{O(1)}\right)$ many states. Projection onto the tracks corresponding to the variables $x_{1}, \ldots, x_{n}$ results again into an automaton with $\exp \left(1,|\psi|^{O(1)}\right)$ many states. Hence, for $\neg \exists y_{1} \cdots \exists y_{m}: \neg \varphi$ there exists an $n$-dimensional automaton with $\exp \left(2,|\psi|^{O(1)}\right)$ many states. But, we do not need to construct this automaton explicitly but only have to check emptiness of its language, which can be done on the fly in exponential space.

For the lower bound, we reuse our construction from the proof of Theorem 4.2, We start with an $\exp (1, n)$-space-bounded machine $M$ that accepts an EXPSPACE-complete language. We carry out the same construction as in the proof of Theorem 4.2, but replace $2^{m}$ (resp. $m$ ) everywhere by $m$ (resp. the input length $n$ ). In addition, we need the following (trivial) analogue of Lemma 4.1; Let the signature $\mathcal{S}$ contain a binary symbol $r$. From a given number $n$ (encoded unary), we can construct in linear time a $\Sigma_{1}$-formula $\varphi^{(n)}(x, y)$ such that for every $\mathcal{S}$-structure $\mathcal{A}$ and all elements $a, b \in \mathcal{A}$ we have: $(a, b) \in r^{n}$ if and only if $\mathcal{A}=\varphi^{(n)}(a, b)$.

Then, the final formula from the proof of Theorem 4.2 can be written as

$$
\exists x \in U_{0}\left\{\begin{array}{l}
\exists y_{1}, y_{2}, y_{3}\left(\delta\left(x, y_{1}\right) \wedge \sigma_{0}^{(n)}\left(y_{1}, y_{2}\right) \wedge \sigma_{\Omega}\left(y_{2}, y_{3}\right) \wedge y_{3} \in U_{1}\right) \wedge \\
\forall y\left(\neg \mu^{(n)}(x, y) \vee y \in U_{2}\right) \wedge \\
\exists y_{0}, z_{0}, \ldots, y_{n+1}, z_{n+1}\left(\iota_{0,1}^{(n)}\left(x, y_{0}\right) \wedge \iota_{q_{0}}\left(y_{0}, z_{0}\right) \wedge \bigwedge_{i=1}^{n+1} \iota_{0,1}^{(n)}\left(z_{i-1}, y_{i}\right) \wedge \iota_{a_{i}}\left(y_{i}, z_{i}\right)\right)
\end{array}\right\} .
$$

This formula is equivalent to a $\Sigma_{2}$-formula. Moreover, this formula is true in the string automatic structure $\mathcal{A}$ (of bounded degree) from the proof of Theorem 4.2, if and only if the input $w=a_{1} a_{2} \cdots a_{n}$ is accepted by the machine $M$.

As before, Theorems 5.15 .3 can be extended to tree automatic structures as follows:

Theorem 5.4. The following holds:

1. The $\Sigma_{1}$-model checking problem $\Sigma_{1}$-FOMC(TA) for all tree automatic presentations is in EXPTIME.

2. There exists a fixed tree automatic structure of bounded degree and linear growth with an EXPTIME-complete $\Sigma_{1}$-theory.

3. The $\Sigma_{2}$-model checking problem $\Sigma_{2}$-FOMC(TA) for all tree automatic presentations is in 2EXPTIME.

4. There exists a tree automatic structure of bounded degree with a 2EXPTIME-complete $\Sigma_{2}$ theory. 


\section{Open problems}

The most obvious open question regards the uniform first-order theory for (injective) tree automatic structures: we do not know whether it is 4EXPTIME-hard. Moreover, we don't know an upper bound for the uniform first-order theory for arbitrary tree automatic structures. The reason is that we do not know the complexity of transforming such a presentation into an equivalent injective one (which is possible by [7]).

In [519], it is shown that not only the first-order theory of every string automatic structure is (uniformly) decidable, but even its extension by the quantifiers "there are infinitely many $x$ with ..." and "the number of $x$ satisfying ... is divisible by $p$ ". In [22], we proved that this extended theory can be decided in triply exponential time for $(\omega)$-automatic structures of bounded degree. It is not clear whether the doubly-exponential upper bound proved in this paper extends to this more expressive theory.

Recall that there are tree automatic structures which are not string automatic. Provided 2 EXPSPACE $\neq 3$ EXPTIME, our results on the non-uniform first-order theories imply the existence of such a structure of bounded degree (namely the tree automatic structure constructed in the proof of Theorem 4.3). But no example is known that does not rest on the complexity theoretic assumption 2EXPSPACE $\neq 3$ EXPTIME.

For $n \geq 3$, the precise complexity of the $\Sigma_{n}$-theory of a string/tree automatic structure of bounded degree remains open. We know that these theories belong to 2EXPSPACE for string automatic structures and to 3EXPTIME for tree automatic structures. Moreover, from our results for the $\Sigma_{2}$-fragment we obtain lower bounds of EXPSPACE and 2EXPTIME, respectively.

Conjecture 6.1. For every $n \geq 3$, the problems $\Sigma_{n}$-FOMC(SAb) and $\Sigma_{n}$-FOMC(TAb) belong to EXPSPACE and 2EXPTIME, respectively.

A possible attack to this conjecture would follow the line of argument in the proof of Theorem [3.6 and would therefore be based on Gaifman's theorem. To make this work, the exponential bound in Gaifman's theorem would have to be reduced which leads to the following conjecture.

Conjecture 6.2. Let $\mathcal{A}$ be a structure, $\left(a_{1}, \ldots, a_{k}\right),\left(b_{1}, \ldots, b_{k}\right) \in \mathcal{A}^{k}, d \geq 0$, and $D_{1}, \ldots, D_{k} \geq$ $d \cdot 2^{n}$ such that

$$
\left(\mathcal{A} \uparrow\left(\bigcup_{i=1}^{k} S\left(D_{i}, a_{i}\right)\right), a_{1}, \ldots, a_{k}\right) \simeq\left(\mathcal{A} \uparrow\left(\bigcup_{i=1}^{k} S\left(D_{i}, b_{i}\right)\right), b_{1}, \ldots, b_{k}\right) .
$$

Then, for every $\Sigma_{n}$-formula $\varphi\left(x_{1}, \ldots, x_{k}\right)$ of quantifier depth at most $d$, we have:

$$
\mathcal{A} \models \varphi\left(a_{1}, \ldots, a_{k}\right) \Longleftrightarrow \mathcal{A} \models \varphi\left(b_{1}, \ldots, b_{k}\right) .
$$

\section{References}

1. V. Bárány. Invariants of automatic presentations and semi-synchronous transductions. In STACS'06, Lecture Notes in Comp. Science vol. 3884, pages 289-300. Springer, 2006.

2. V. Bárány, Ł. Kaiser, and S. Rubin. Cardinality and counting quantifiers on omega-automatic structures. In STACS'08, pages 385-396. IFIB Schloss Dagstuhl, 2008.

3. M. Benedikt, L. Libkin, T. Schwentick, and L. Segoufin. Definable relations and first-order query languages over strings. J. ACM, 50(5):694-751, 2003. 
4. A. Blumensath. Automatic structures. Technical report, RWTH Aachen, 1999.

5. A. Blumensath and E. Grädel. Automatic Structures. In LICS'00, pages 51-62. IEEE Computer Society Press, 2000.

6. A. K. Chandra, D. C. Kozen, and L. J. Stockmeyer. Alternation. J. ACM, 28(1):114-133, 1981.

7. T. Colcombet and C. Löding. Transforming structures by set interpretations. Logical Methods in Computer Science, 3:1-36, 2007.

8. H. Comon, M. Dauchet, R. Gilleron, C. Löding, F. Jacquemard, D. Lugiez, S. Tison, and M. Tommasi. Tree automata techniques and applications. Available on: http://www.grappa.univ-lille3.fr/tata, 2007. Release October, 12th 2007.

9. K. Compton and C. Henson. A uniform method for proving lower bounds on the computational complexity of logical theories. Annals of Pure and Applied Logic, 48:1-79, 1990.

10. C. Delhommé, V. Goranko, and T. Knapik. Automatic linear orderings. Manuscript, 2003.

11. C. Elgot. Decision problems of finite automata design and related arithmetics. Trans. Am. Math. Soc., 98:21-51, 1961.

12. D. Epstein, J. Cannon, D. Holt, S. Levy, M. Paterson, and W. Thurston. Word Processing In Groups. Jones and Bartlett Publishers, Boston, 1992.

13. H. Gaifman. On local and nonlocal properties. In J. Stern, editor, Logic Colloquium '81, pages 105-135. North-Holland, 1982.

14. B. Hodgson. On direct products of automaton decidable theories. Theoretical Computer Science, 19:331335, 1982.

15. I. Ishihara, B. Khoussainov, and S. Rubin. Some results on automatic structures. In LICS'02, pages 235-244. IEEE Computer Society Press, 2002.

16. B. Khoussainov and A. Nerode. Automatic presentations of structures. In Logic and Computational Complexity, Lecture Notes in Comp. Science vol. 960, pages 367-392. Springer, 1995.

17. B. Khoussainov and S. Rubin. Graphs with automatic presentations over a unary alphabet. J. Autom. Lang. Comb., 6:467-480, 2001.

18. B. Khoussainov, S. Rubin, and F. Stephan. On automatic partial orders. In LICS'03, pages 168-177. IEEE Computer Society Press, 2003.

19. B. Khoussainov, S. Rubin, and F. Stephan. Definability and regularity in automatic structures. In STACS'04, Lecture Notes in Comp. Science vol. 2996, pages 440-451. Springer, 2004.

20. D. Kuske. Is Cantor's theorem automatic? In LPAR'03, Lecture Notes in Comp. Science vol. 2850, pages 332-345. Springer, 2003.

21. D. Kuske and M. Lohrey. Euler paths and ends in automatic and recursive graphs. In Automata and Formal Languages 2008, pages 245-256. Hungarian Academy of Sciences, 2008.

22. D. Kuske and M. Lohrey. First-order and counting theories of $\omega$-automatic structures. Journal of Symbolic Logic, 73:129-150, 2008.

23. D. Kuske and M. Lohrey. Hamiltonicity of automatic graphs. In G. Ausiello, J. Karhumäki, G. Mauri, and L. Ong, editors, IFIP-TCS'08, pages 445-459. Springer, 2008.

24. M. Lohrey. Automatic structures of bounded degree. In LPAR'03, Lecture Notes in Comp. Science vol. 2850, pages 344-358. Springer, 2003.

25. A. Meyer. Weak monadic second order theory of one successor is not elementary recursive. In Proc. Logic Colloquium, Lecture Notes in Mathematics vol. 453, pages 132-154. Springer, 1975.

26. C. H. Papadimitriou. Computational Complexity. Addison Wesley, 1994.

27. S. Rubin. Automata presenting structures: A survey of the finite string case. Bulletin of Symbolic Logic, 14:169-209, 2008.

28. H. Seidl. Single-valuedness of tree transducers is decidable in polynomial time. Theoretical Computer Science, 106:135-181, 1992.

29. P. V. Silva and B. Steinberg. A geometric characterization of automatic monoids. The Quarterly Journal of Mathematics, 55:333-356, 2004.

30. A. Weber. On the valuedness of finite transducers. Acta Informatica, 27:749-780, 1990. 